\title{
STUDIES OF MILLIMETER-WAVE ATMOSPHERIC NOISE ABOVE MAUNA KEA
}

\author{
J. Sayers ${ }^{1,6}$, S. R. Golwala ${ }^{2}$, P. A. R. Ade ${ }^{3}$, J. E. Aguirre ${ }^{3,4}$, J. J. Bock ${ }^{1}$, S. F. Edgington ${ }^{2}$, J. Glenn ${ }^{5}$, A. Goldin ${ }^{1}$, D. Haig ${ }^{3}$,

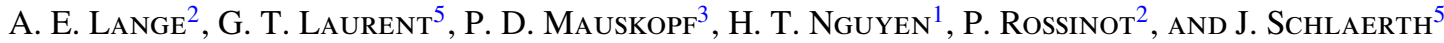 \\ ${ }^{1}$ Jet Propulsion Laboratory, California Institute of Technology, 4800 Oak Grove Drive, Pasadena, CA 91109, USA; jack@ caltech.edu \\ ${ }^{2}$ Division of Physics, Mathematics, \& Astronomy, California Institute of Technology, Mail Code 59-33, Pasadena, CA 91125, USA \\ ${ }^{3}$ Physics and Astronomy, Cardiff University, 5 The Parade, P. O. Box 913, Cardiff CF24 3YB, Wales, UK \\ ${ }^{4}$ University of Pennsylvania, 209 South 33rd Street, Philadelphia, PA 19104, USA \\ ${ }^{5}$ Center for Astrophysics and Space Astronomy \& Department of Astrophysical and Planetary Sciences, University of Colorado, 389 UCB, Boulder, CO 80309 , USA \\ Received 2009 April 24; accepted 2009 November 24; published 2009 December 23
}

\begin{abstract}
We report measurements of the fluctuations in atmospheric emission (atmospheric noise) above Mauna Kea recorded with Bolocam at 143 and $268 \mathrm{GHz}$ from the Caltech Submillimeter Observatory. The $143 \mathrm{GHz}$ data were collected during a 40 night observing run in late 2003 , and the $268 \mathrm{GHz}$ observations were made in early 2004 and early 2005 over a total of 60 nights. Below $\simeq 0.5 \mathrm{~Hz}$, the data time-streams are dominated by atmospheric noise in all observing conditions. The atmospheric noise data are consistent with a Kolmogorov-Taylor turbulence model for a thin wind-driven screen, and the median amplitude of the fluctuations is $280 \mathrm{mK}^{2} \mathrm{rad}^{-5 / 3}$ at $143 \mathrm{GHz}$ and $4000 \mathrm{mK}^{2} \mathrm{rad}^{-5 / 3}$ at $268 \mathrm{GHz}$. Comparing our results with previous ACBAR data, we find that the normalization of the power spectrum of the atmospheric noise fluctuations is a factor of $\simeq 80$ larger above Mauna Kea than above the South Pole at millimeter wavelengths. Most of this difference is due to the fact that the atmosphere above the South Pole is much drier than the atmosphere above Mauna Kea. However, the atmosphere above the South Pole is slightly more stable as well: the fractional fluctuations in the column depth of precipitable water vapor are a factor of $\simeq \sqrt{2}$ smaller at the South Pole compared to Mauna Kea. Based on our atmospheric modeling, we developed several algorithms to remove the atmospheric noise, and the best results were achieved when we described the fluctuations using a low-order polynomial in detector position over the $8^{\prime}$ field of view. However, even with these algorithms, we were not able to reach photon-background-limited instrument photometer performance at frequencies below $\simeq 0.5 \mathrm{~Hz}$ in any observing conditions. We also observed an excess low-frequency noise that is highly correlated between detectors separated by $\lesssim(f / \#) \lambda$; this noise appears to be caused by atmospheric fluctuations, but we do not have an adequate model to explain its source. We hypothesize that the correlations arise from the classical coherence of the electromagnetic field across a distance of $\simeq(f / \#) \lambda$ on the focal plane.
\end{abstract}

Key words: atmospheric effects - site testing - techniques: photometric

Online-only material: color figures

\section{INTRODUCTION}

A number of wide-field ground-based millimeter/ submillimeter imaging arrays have been commissioned during the past 15 years, including SCUBA (Holland et al. 1999), MAMBO (Kreysa et al. 1998), Bolocam (Glenn et al. 1998), SHARC II (Dowell et al. 2003), APEX-SZ (Dobbs et al. 2006), LABOCA (Kreysa et al. 2003), ACT (Kosowsky 2003), and SPT (Ruhl et al. 2004). Since these cameras are operated at ground-based telescopes, they all see emission from water vapor in the atmosphere. In almost all cases, the raw data from these cameras are dominated by atmospheric noise caused by fluctuations in this emission. ${ }^{7}$ All of these cameras make use of the fact that the atmospheric water vapor is in the near field, and therefore most of the fluctuations in the atmospheric emission are recorded as a common-mode signal among all of the detectors (Jenness et al. 1998; Borys et al. 1999; Reichertz et al.

\footnotetext{
6 NASA Postdoctoral Program Fellow.

7 The column depth of oxygen in the atmosphere also produces a non-negligible amount of emission, a factor of a few less than the emission from water vapor under typical conditions at Mauna Kea. However, the oxygen in the atmosphere is well mixed, and therefore fluctuations in the emission are minimal. In contrast, the temperature of the atmosphere tends to be close to the condensation point of the water vapor, and causes the water vapor to be poorly mixed in the atmosphere. Therefore, there are, in general, significant fluctuations in the emission from water vapor (Masson 1994).
}

2001; Weferling et al. 2002; Archibald et al. 2002). Most of the atmospheric noise can be removed from the data by subtracting this common-mode signal, and this method has been shown to be at least as effective as the traditional beam-switching or chopping techniques (Conway et al. 1965; Weferling et al. 2002; Archibald et al. 2002).

However, this subtraction does not allow recovery of photon-background-limited instrument photometer (BLIP) performance on scales where the atmospheric signal is largest (i.e., at low frequencies in the time-stream data). In the case of Bolocam, the majority of the atmospheric fluctuations can be removed by subtraction of the common-mode signal; but the residual atmospheric noise still limited the sensitivity of our data, thus motivating further study of these atmospheric fluctuations. This study focused on two main topics: (1) determining the phenomenology of the atmospheric noise (i.e., could it be modeled in a simple and robust way); and (2) finding more effective ways to remove the atmospheric noise based on this modeling.

\subsection{Instrument Description}

Bolocam is a large format, millimeter-wave camera designed to be operated at the Caltech Submillimeter Observatory (CSO), and $\simeq 115$ optical detectors were used for the observations described in this paper. Cylindrical waveguides and a metal- 
mesh filter are used to define the passbands for the detectors, which can be centered at either 143 or $268 \mathrm{GHz}$ with a $\simeq 15 \%$ fractional bandwidth. Note that, for either configuration, the entire focal plane uses the same passband. A cold (4 K) Lyot stop is used to define the illumination of the $10.4 \mathrm{~m}$ primary mirror with a diameter of $\simeq 8$ meters, and the resulting far-field beams have full width at half-maximums (FWHMs) of $60^{\prime \prime}$ or $30^{\prime \prime}$ (143 or $268 \mathrm{GHz}$ ). The detector array, which utilizes silicon nitride micromesh (spider-web) bolometers (Mauskopf et al. 1997), has a hexagonal geometry with nearby detectors separated by $40^{\prime \prime}$, and the field of view (FOV) is approximately $8^{\prime}$.

The optical efficiency from the cryostat window to the detectors is $8 \%$ at $143 \mathrm{GHz}$ and $19 \%$ at $268 \mathrm{GHz}$; at each frequency approximately half of the loss in efficiency is due to coupling to the Lyot stop and half is due to inefficiencies (reflection, standing waves, or loss) in the metal-mesh filter stack. At $143 \mathrm{GHz}$, the typical optical load from the atmosphere is relatively small $(\simeq 0.5 \mathrm{pW}$ or $10 \mathrm{~K})$, but the total optical load is $\simeq 4 \mathrm{pW}(80 \mathrm{~K})$, most of which is sourced by warm surfaces inside the relay optics box. The atmosphere contributes an optical load of 5-15 pW (20-60 K) per detector at $268 \mathrm{GHz}$, and there is an additional load of $\simeq 10 \mathrm{pW}(40 \mathrm{~K})$ due to the warm and cold optics. Optical shot and Bose noise contribute in roughly equal amounts to the total photon noise at each observing frequency, with the BLIP NEP $\gamma \simeq 1.5 \mathrm{mK} / \sqrt{\mathrm{Hz}}\left(2.3 \mathrm{mK}_{\mathrm{CMB}} / \sqrt{\mathrm{Hz}}\right)$ at $143 \mathrm{GHz}$ and the BLIP NEP $\gamma \simeq 0.8 \mathrm{mK} / \sqrt{\mathrm{Hz}}\left(4.5 \mathrm{mK}_{\mathrm{CMB}} /\right.$ $\sqrt{\mathrm{Hz}}$ ) at $268 \mathrm{GHz}{ }^{8}$ More details of the Bolocam instrument can be found in Glenn et al. (1998); Glenn et al. (2003); Haig et al. (2004); and Sayers (2007).

The data we describe in this paper were collected during three separate observing runs at the CSO: a 40 night run at $143 \mathrm{GHz}$ in late 2003, a 10 night run at $268 \mathrm{GHz}$ in early 2004 , and a 50 night run at $268 \mathrm{GHz}$ in early 2005 . For the $143 \mathrm{GHz}$ observations, we focused on two science fields, one centered on the Lynx field at $08^{\mathrm{h}} 49^{\mathrm{m}} 12^{\mathrm{s}},+44^{\mathrm{d}} 50^{\mathrm{m}} 24^{\mathrm{s}}(\mathrm{J} 2000)$ and one coinciding with the Subaru/XMM-Newton Deep Survey (SXDS or SDS1) centered at $02^{\mathrm{h}} 18^{\mathrm{m}} 00^{\mathrm{s}},-5^{\mathrm{d}} 00^{\mathrm{m}} 00^{\mathrm{s}}(\mathrm{J} 2000)$. The $268 \mathrm{GHz}$ observations were all focused on the COSMOS field at $10^{\mathrm{h}} 00^{\mathrm{m}} 29^{\mathrm{s}},+2^{\mathrm{d}} 12^{\mathrm{m}} 21^{\mathrm{s}}(\mathrm{J} 2000)$. All three of these fields are blank, which means they contain very little astronomical signal. Therefore, our data are well suited to measure the signal caused by emission from the atmosphere. To map these fields, we raster-scanned the telescope parallel to the R.A. or decl. axis at $4 \operatorname{arcmin~s}{ }^{-1}$ for the $143 \mathrm{GHz}$ observations and $2 \operatorname{arcmin~s}{ }^{-1}$ for the $268 \mathrm{GHz}$ observations. ${ }^{9}$ Throughout this paper, we will refer to single scans and single observations; a scan is one raster across the field and is $\simeq 15 \mathrm{~s}\left(\simeq 30^{\prime}-60^{\prime}\right)$ in length and an observation is a set of $\simeq 15-20$ scans that completely map the science field, which takes $\simeq 10$ minutes. Our total data set contains approximately 1000 observations at each observing frequency, with the $143 \mathrm{GHz}$ data split evenly among Lynx and

\footnotetext{
8 The subscript cosmic microwave background (CMB) is used throughout this paper to denote CMB temperatures; all temperatures given without a subscript refer to Rayleigh-Jeans temperatures.

9 Slower scan speeds improve our observing efficiency by reducing the fractional amount of time spent turning the telescope around between scans (the CSO turnaround time is approximately $10 \mathrm{~s}$ regardless of scan speed), but faster scan speeds improve the instantaneous sensitivity of the camera by moving the signal band to higher frequencies where there is less atmospheric noise. Several scan speeds were tried at each observing frequency to find the best combination of observing efficiency and instantaneous sensitivity, and we found that $4 \operatorname{arcmin~s}^{-1}$ is optimal for $143 \mathrm{GHz}$ observations and $2 \operatorname{arcmin~s}^{-1}$ is optimal for $268 \mathrm{GHz}$ observations. Note that it may be possible to optimize the CSO telescope drive servo to improve the turnaround time, but this has not been attempted.
}

SDS1. Flux calibration was determined from observations of Uranus, Neptune, and Mars, and nearby quasars were used for pointing reconstruction. A more detailed description of the data is given in Sayers et al. (2009) and J. E. Aguirre et al. (2010, in preparation).

\subsection{Typical Observing Conditions}

Since atmospheric noise from water vapor is generally the limiting factor in the sensitivity of broadband, ground-based, millimeter-wave observations, the premier sites for these observations, which include Mauna Kea, Atacama, and the South Pole, are extremely dry. On Mauna Kea, the CSO continuously monitors the atmospheric opacity with a narrowband, heterodyne $\tau$-meter that measures the optical depth at $225 \mathrm{GHz}\left(\tau_{225}\right)$ (Chamberlin 2004). Since $\tau_{225}$ is a monotonically increasing function of the column depth of precipitable water vapor in the atmosphere, these $\tau_{225}$ measurements can be used to quantify the dryness of the atmosphere above Mauna Kea. Historically, the median value of $\tau_{225}$ is 0.091 during winter nights, which corresponds to a column depth of precipitable water vapor of $\mathcal{C}_{P W}=1.68 \mathrm{~mm}$ (Pardo et al. 2001a, 2001b, 2005; Figure 1). The 25th and 75th centiles at Mauna Kea are 1.00 and $2.92 \mathrm{~mm}$, respectively. Note that the 25th, 50th, and 75th centiles of our data sets closely match these historical averages, so our data are a fair representation of the average conditions on Mauna Kea. For comparison, the median value of $\mathcal{C}_{P W}$ at the ALMA site in Atacama is $\simeq 1.00 \mathrm{~mm}$ during winter nights, while the median value at the South Pole is around $0.25 \mathrm{~mm}$ during the winter (Radford \& Chamberlin 2000; Lane 1998; Peterson et al. 2003; Stark et al. 2001). ${ }^{10}$

\section{KOLMOGOROV-TAYLOR (K-T)/THIN-SCREEN ATMOSPHERIC MODEL}

The K-T model of turbulence provides a good description of air movement in the atmosphere (Kolmogorov 1941; Taylor 1938; Tatarskii 1961). According to the model, processes such as convection and wind shear inject energy into the atmosphere on large length scales, of order several kilometers (Kolmogorov 1941; Wright 1996). This energy is transferred to smaller scales by eddy currents, until it is dissipated by viscous forces at Kolmogorov microscales, corresponding to the smallest scales in turbulent flow and of order several millimeters for the atmosphere (Kolmogorov 1941). For a three-dimensional volume, the model predicts a power spectrum for the fluctuations from this turbulence that is proportional to $|\vec{q}|^{-11 / 3}$, where $\vec{q}$ is a three-dimensional spatial frequency with units of $1 /$ length. The same spectrum holds for particulates that are passively entrained in the atmosphere, such as water vapor (Tatarskii 1961).

For our analysis, we adopted the two-dimensional thin-screen model described by Lay \& Halverson (2000), and a schematic of this thin-screen model is given in Figure 2. This model assumes that the fluctuations in water vapor occur in a turbulent layer at a height $h_{\text {av }}$ with a thickness $\Delta h$, where $h_{\text {av }} \gg \Delta h$. This layer is moved horizontally across the sky by wind at an angular velocity $\vec{w}$. Given these assumptions and following the notation of Bussmann et al. (2005), the three-dimensional KolmogorovTaylor power spectra reduces to

$$
P(\vec{\alpha})=B_{v}^{2}(\sin \epsilon)^{(1-b)}|\vec{\alpha}|^{-b},
$$

\footnotetext{
${ }^{10}$ Note that the scaling between $\mathcal{C}_{P W}$ and opacity is different at the three sites
} due to the different atmospheric conditions at each location (see Figure 1). 


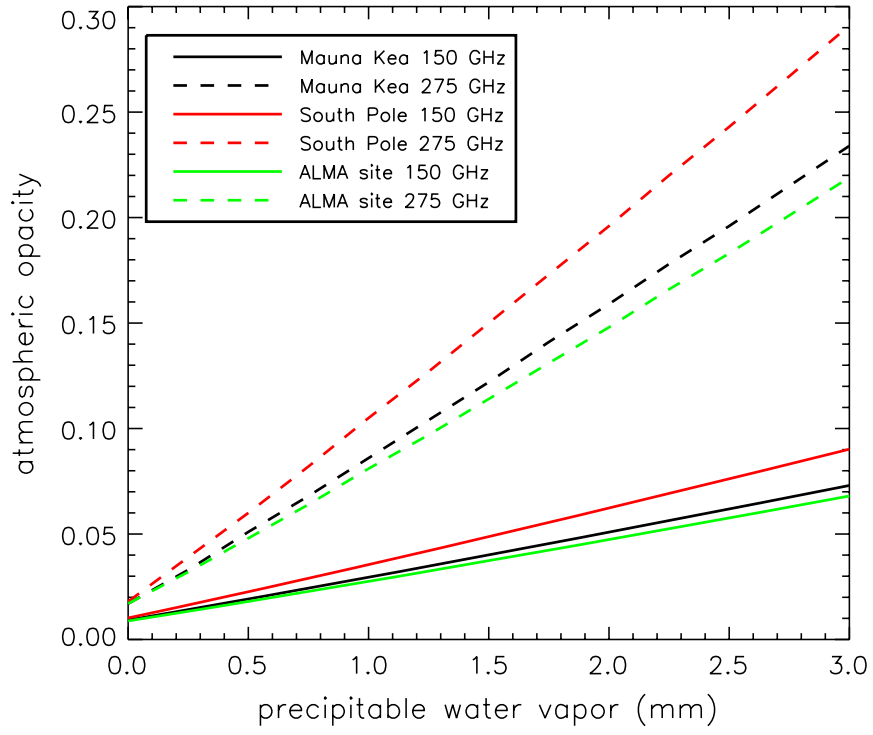

Figure 1. Atmospheric opacity as a function of $\mathcal{C}_{P W}$ for Mauna Kea, the South Pole, and the ALMA site. The opacity is shown at $150 \mathrm{GHz}$ and $275 \mathrm{GHz}$, the approximate centers of the Bolocam/ACBAR observing bands. All of the scalings were derived using the Pardo ATM algorithm (Pardo et al. 2001a, 2001b, 2005).

(A color version of this figure is available in the online journal.)

where $B_{v}^{2}$ is the amplitude of the power spectrum at zenith, $\epsilon$ is the elevation angle of the telescope, $\vec{\alpha}$ is the two-dimensional angular frequency with units of $1 /$ radians, and $b$ is the power law of the model (equal to $11 / 3$ for the $\mathrm{K}-\mathrm{T}$ model). Note that $B_{v}^{2}$ has units of $\mathrm{mK}^{2} \operatorname{rad}^{-5 / 3}$ for $b=11 / 3$.

\section{FITTING BOLOCAM DATA TO THE K-T THEORY}

\subsection{Calculating the Wind Velocity}

If the angular wind velocity, $\vec{w}$, is assumed to be constant and the spatial structure of the turbulent layer is static on the timescales required for the wind to move the layer past our beams (Taylor 1938), then detectors aligned with the angular wind velocity will see the same atmospheric emission, but at different times (Church 1995). Making reasonable assumptions for the wind speed $\left(10 \mathrm{~m} \mathrm{~s}^{-1}\right)$ and height of the turbulent layer $(1 \mathrm{~km})$ yields an angular speed of approximately $30 \operatorname{arcmin~s}^{-1}$ for the layer. Note that this is much faster than our maximum scan speed of $4 \operatorname{arcmin~s}^{-1}$. Since the diameter of the Bolocam focal plane is $8^{\prime}$, the angular wind velocity and spatial structures only need to be stable for a fraction of a second to make our assumption valid. To look for these time-lagged correlations, we computed the relative cross power spectrum between every pair of bolometers, described by

$$
x \operatorname{PSD}_{i, j}\left(f_{m}\right)=\frac{D_{i}\left(f_{m}\right)^{*} D_{j}\left(f_{m}\right)}{\sqrt{\left|D_{i}\left(f_{m}\right)\right|^{2}} \sqrt{\left|D_{j}\left(f_{m}\right)\right|^{2}}},
$$

where $x \operatorname{PSD}_{i, j}\left(f_{m}\right)$ is the relative cross power spectral density (PSD) between bolometers $i$ and $j, D_{i}\left(f_{m}\right)$ is the Fourier transform of the data time-stream for bolometer $i$ at Fourier space sample $m$, and $f_{m}$ is the frequency (in $\mathrm{Hz}$ ) of sample $m$.

If two bolometers see the same signal at different times, then the cross PSD of these bolometers will have a phase angle described by

$$
\tan ^{-1}(x \mathrm{PSD})=\Theta_{f}=2 \pi f \Delta t,
$$

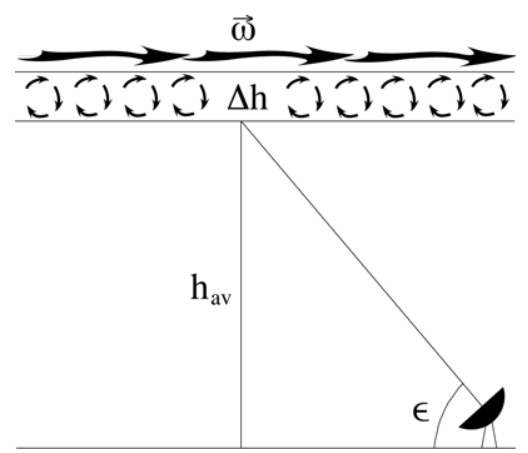

Figure 2. Diagram of the thin-screen turbulence model described by Lay \& Halverson (2000) that is used throughout this paper.

where $f$ is the frequency in $\mathrm{Hz}$ and $\Delta t$ is the time difference (in s) between the signal recorded by the two bolometers. Therefore, the slope of a linear fit to $\Theta_{f}$ versus $f$ will be proportional to $\Delta t$. If the simple atmospheric model that we have assumed is correct, then $\Delta t / \theta_{\text {pair }}$ should be a sinusoidally varying function of the relative angle on the focal plane between the bolometer pair, $\phi_{\text {pair }}$, where $\theta_{\text {pair }}$ is the angular separation of the two bolometers (i.e., if one bolometer is located at position $\left(x_{1}, y_{1}\right)$ and another bolometer is located at position $\left(x_{2}, y_{2}\right)$, then $\phi_{\text {pair }}=\tan ^{-1}\left(\frac{y_{2}-y_{1}}{x_{2}-x_{1}}\right)$ and $\left.\theta_{\text {pair }}=\sqrt{\left(x_{2}-x_{1}\right)^{2}+\left(y_{2}-y_{1}\right)^{2}}\right)$. Some examples of $2 \pi \Delta t / \theta_{\text {pair }}$ versus $\phi_{\text {pair }}$ are given in Figure 3. In general, the model provides an excellent fit for roughly half of our data (typically the data collected in better weather as quantified by the time-stream rms). The remaining data tend to contain several outliers and/or features in addition to the underlying sinusoid given by the model.

The model fits also provide an estimate of the angular wind speed, with

$$
|\vec{w}|=\theta_{\text {pair }} / \Delta t
$$

where $\theta_{\text {pair }} \simeq 40^{\prime \prime}$ for adjacent detectors on the Bolocam focal plane. Histograms showing the angular wind speed for all of our observations at both 143 and $268 \mathrm{GHz}$ are given in Figure 4. Note that the median angular wind speed is $31 \operatorname{arcmin~s}^{-1}$ for the $143 \mathrm{GHz}$ data and $35 \mathrm{arcmin} \mathrm{s}^{-1}$ for the $268 \mathrm{GHz}$ data, which is approximately what we expected for a physically reasonable model of the atmosphere.

\subsection{Instantaneous Correlations}

Equation (1) can be converted from a power spectrum in angular frequency space to a correlation function as a function of angular separation. Since the power spectrum is azimuthally symmetric, we can write $P(\vec{\alpha})$ as $P(\alpha)$, where $\alpha=|\vec{\alpha}|$. This power spectrum will produce a correlation function according to

$$
C(\theta)=2 \pi \int_{\alpha_{\min }}^{\infty} d \alpha \alpha P(\alpha) J_{0}(2 \pi \alpha \theta),
$$

where $\theta$ is the angular separation in radians, $\alpha_{\min }$ is the maximum length scale of the turbulence, and $J_{0}$ is the zerothorder Bessel function of the first kind.

To compare our data to this model, we calculated the correlation between the time-streams of every bolometer pair according to

$$
C_{i j}=\frac{1}{N} \sum_{n} d_{i n} d_{j n},
$$

where $C_{i j}$ is the correlation between bolometer $i$ and bolometer $j$ in $\mathrm{mK}^{2}, N$ is the number of time-stream samples, and $d_{i n}$ is 

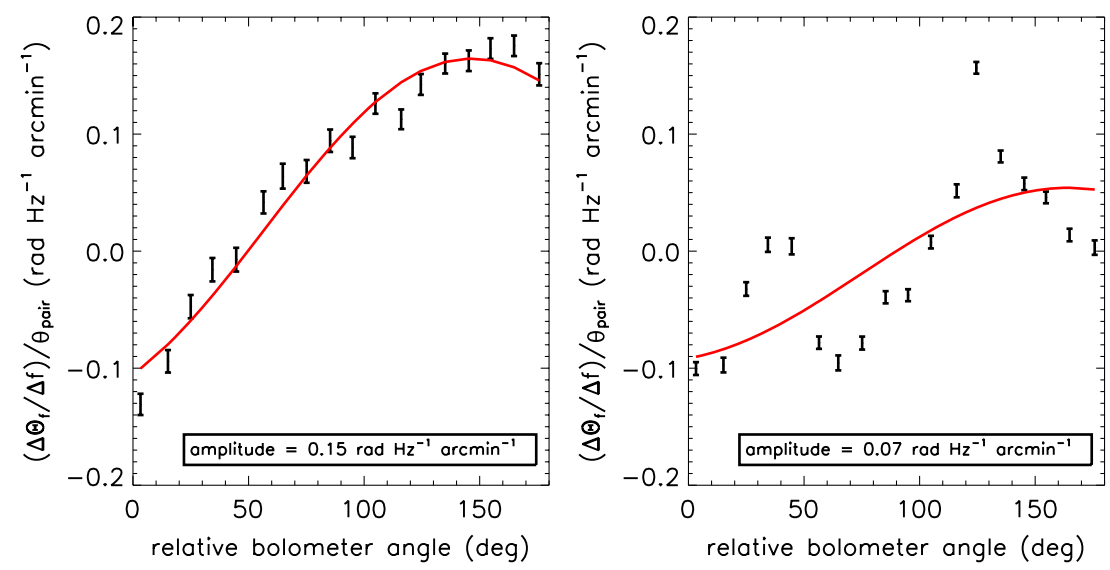

Figure 3. Plots of $\frac{\left(\Delta \Theta_{f} / \Delta f\right)}{\theta_{\text {pair }}}$ averaged over all bolometer pairs and all scans for a single observation. This slope is binned according to $\phi_{\text {pair }}$, and the sinusoidal fit predicted from the thin-screen $\mathrm{K}-\mathrm{T}$ model is overlaid in red. In general, roughly half of our data are well described by this model, with a typical example shown in the left-hand plot. The other half of the data tend to contain outliers and/or additional features; the right-hand plot shows an example of one of these data sets.

(A color version of this figure is available in the online journal.)

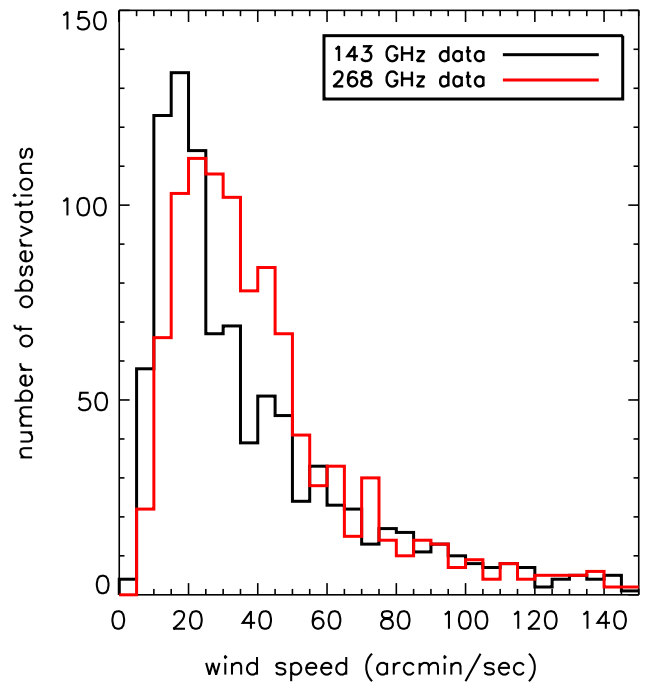

Figure 4. Angular wind speed of the turbulent layer for every observation at both $143 \mathrm{GHz}$ and $268 \mathrm{GHz}$. Note that the median value of the distributions is 31 and $35 \operatorname{arcmin~s}^{-1}$, respectively. This corresponds to a linear speed of $10 \mathrm{~m} \mathrm{~s}^{-1}$ if the layer is at a height of $1 \mathrm{~km}$, which is physically reasonable. (A color version of this figure is available in the online journal.)

the time-stream data for bolometer $i$ at time sample $n$. A single correlation value for each pair was calculated for each $\simeq 15$-slong scan made while observing one of the science fields, and then averaged over the twenty scans in one complete observation of the field. Therefore, we have assumed that the atmospheric noise conditions do not change over the $\simeq 10$-minute-long observation and are independent of the scan direction, which is reasonable given that the typical angular wind speed is much larger than our scan speed. The $C_{i j}$ were then binned as a function of angular separation between bolometer $i$ and bolometer $j$ to give correlation as a function of $\theta$.

Ideally, we would like to compare our data directly to the theoretical model using Equation (2). However, evaluating the integral in Equation (2) is non-trivial, especially when the effects of Bolocam's finite beams, data processing, etc., are included. Therefore, we have determined the theoretical correlation function based on the $\mathrm{K}-\mathrm{T}$ model via simulation. First, we generate 50 two-dimensional projections (i.e., maps) of the atmospheric fluctuation signal according to the power spectrum given in Equation (1). In each of these realizations, the phases of the different spatial frequency components are taken to be random. Next, we convolve each map with the profile of a Bolocam beam. ${ }^{11}$ Then, we generate time-stream data by moving the atmospheric fluctuation map across our detector array at a rate given by the angular wind speed we calculated in Section 3.1. These simulated time-streams are then processed in the same way as our real data, including removing the mean signal level from each $\simeq 15$-s-long scan. Finally, we determine the values of $C_{i j}$ for the simulated data, averaging over all 50 realizations, and bin these $C_{i j}$ as a function of bolometer separation.

The shape of the theoretical $C(\theta)$ determined from these simulations will depend not only on the value of the powerlaw index, $b$, but also on the height of the turbulent layer, $h$. Any reasonable value of $h$ will be in the near field for Bolocam, so the physical size of the beam profiles (in meters) will be approximately independent of $h$, which means that the angular size of the beams in the turbulent layer will be a function of $h$. Therefore, a change in the height of the turbulent layer will cause a change in the way that the angular emission profile of the atmosphere is smoothed by the Bolocam beams, which will result in a different profile for $C(\theta)$. Thus, in principle, our measured correlation profiles as a function of separation are sensitive to both $b$ and $h$ (along with $B_{v}^{2}$ ). However, as we explain below and show in Figure 6, we obtain no meaningful constraint on $h$ because our measurement uncertainty on $C(\theta)$ is large compared to the variations in $C(\theta)$ with $h$.

Initially, we assumed that both the height $h$ and the power-law index $b$ were unknown, and ran simulations over a grid of values for each parameter. In our grid, the values of $b$ ran from $2 / 3$ to $20 / 3$ in steps of $1 / 2$, and the values of $h$ were $375,500,750$, $1000,1500,2000,3000,4000$, and $6000 \mathrm{~m}$. Note that we used an irregular step size for $h$ because the beam size is proportional to $1 / h$. Since the computation time required for our simulation is substantial, we were only able to run the full grid of 121 different parameter values over a randomly selected subset of $96143 \mathrm{GHz}$ observations (approximately $10 \%$ of our $143 \mathrm{GHz}$ data). After computing the best-fit value of $B_{v}^{2}$ for each observation and each

\footnotetext{
${ }^{11}$ Since the far-field distance for Bolocam is tens of kilometers, we assume that the atmospheric fluctuations occur in the near field. Therefore, the Bolocam beams can be well approximated by the primary illumination pattern, which is approximately a top hat with a diameter of $8 \mathrm{~m}$. This means that the angular size of the beam will depend on the height of the turbulent layer.
} 

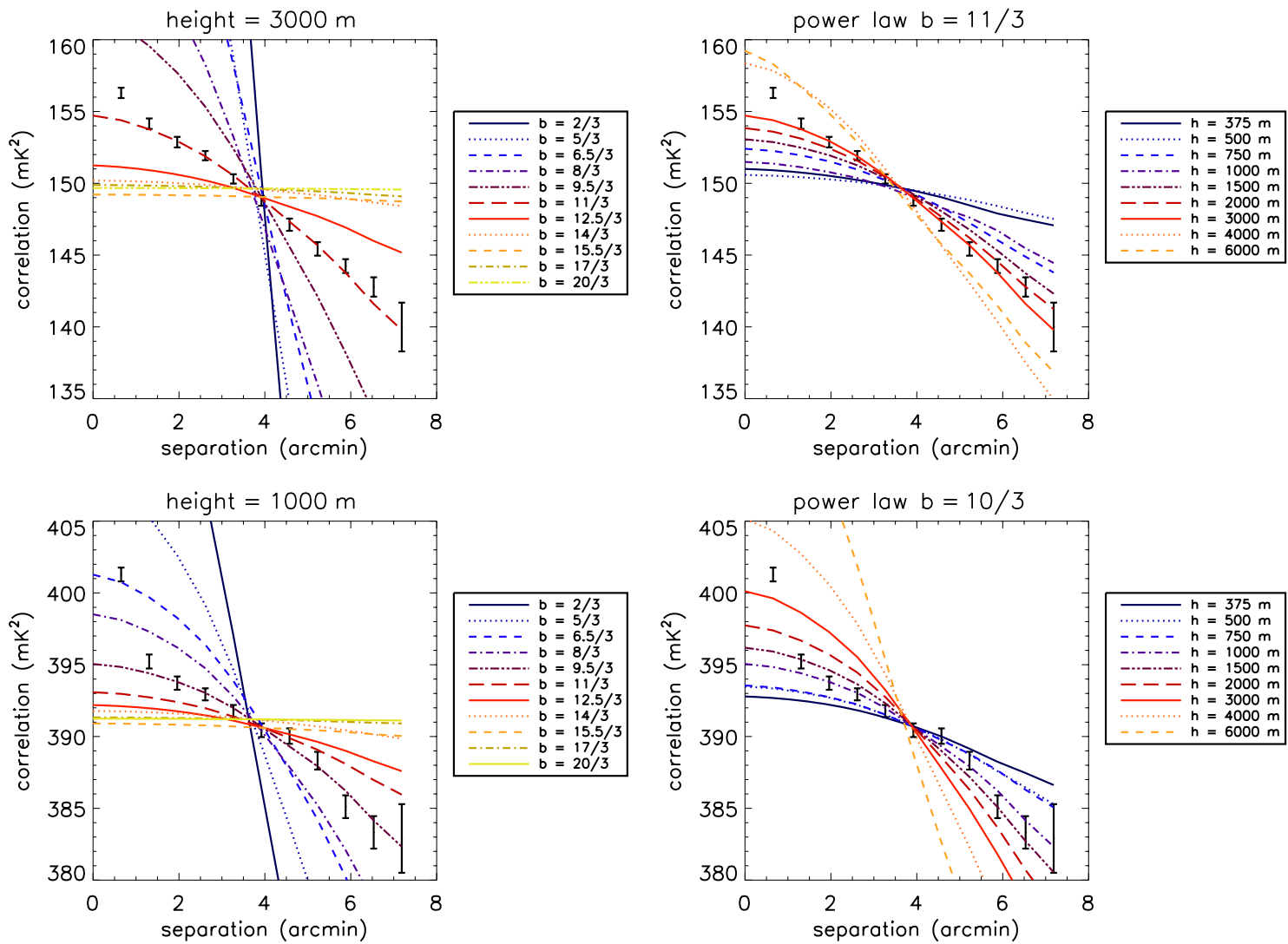

Figure 5. Plots of the average correlation between bolometer pairs as a function of separation between the bolometers. The top row shows data from a $143 \mathrm{GHz}$ observation taken when the amplitude of the atmospheric noise is better than average, and the bottom row shows data from a $143 \mathrm{GHz}$ observation taken when the amplitude of the atmospheric noise is worse than average. The model fits overlaid on the left plots show a range of power-law indices, $b$, at the best-fit value of $h$ for the data set. The model fits overlaid on the right plots show a range of heights, $h$, at the best-fit value of $b$ for the data set. The $\chi^{2}$ value of the model fit for these two observations is similar, and is at roughly the 30 th centile of our complete set of data (i.e., $1 / 3$ of our observations produce a better fit to the $\mathrm{K}-\mathrm{T}$ model, and $2 / 3$ of our observations produce a worse fit to the $\mathrm{K}-\mathrm{T}$ model). Therefore, the quality of the model fit for these observations is fairly typical. Note the degeneracy between $b$ and $h$ in the general shape of the model fits, which makes it difficult to constrain either value precisely for a single observation, especially $h$. These plots clearly show the excess correlation among adjacent bolometers, and note that the adjacent bolometer correlations are discarded when fitting the K-T model to the data.

(A color version of this figure is available in the online journal.)

grid point, we determined what values of $h$ and $b$ provided the best fit to the data. Note that the data from adjacent bolometer pairs are discarded before fitting a model, due to the excess correlations between these pairs (see Section 5.1). Additionally, the constraints on $b$ or $h$ for a single observation are not very precise because there is a wide range of combinations of $b$ and $h$ that will produce very similar model profiles. Some examples of data with model fits overlaid are given in Figure 5. We found the average best-fit value of the power law $b$ is 3.3 with a standard deviation of 1.1, indicating that our data are consistent with the $\mathrm{K}-\mathrm{T}$ model prediction of $b=11 / 3$. Note that Bussmann et al. (2005) previously found the atmosphere above the South Pole to be consistent with the $\mathrm{K}-\mathrm{T}$ model $(b=3.9 \pm 0.6$ when only high signal-to-noise scans are included, $b=4.1 \pm 0.8$ when all scans are included) using ACBAR data that were sensitive to much different physical scales in the atmosphere $(\simeq 1.5 \mathrm{~m}$ beams and $\left.\mathrm{a} \simeq 1^{\circ} \mathrm{FOV}\right) .{ }^{12}$ Figure 6 shows that the best-fit values of $h$ were uniformly distributed over the allowed range, indicating our data do not meaningfully constrain $h$.

\footnotetext{
${ }^{12}$ For ACBAR, the primary mirror is $\simeq 1.5 \mathrm{~m}$ in diameter and adjacent detectors are separated by $16^{\prime}$. As a result, the typical separation between ACBAR beams is larger than the diameter of a single beam as they pass through the water vapor in atmosphere (i.e., each ACBAR beam passes through a different column of atmosphere). In contrast, the $\simeq 10 \mathrm{~m}$ primary at the CSO and $40^{\prime \prime}$ separation between adjacent Bolocam detectors means that there is significant overlap between the beams as they pass through the water vapor in the atmosphere.
}

We have so far assumed that the beams have a top-hat profile while passing through the atmosphere. If the profile is not a top hat and/or varies among pixels, then our simulation will predict a $C(\theta)$ that is too flat. However, given that the data are consistent with the $\mathrm{K}-\mathrm{T}$ model prediction of $b=11 / 3$, there is no indication that such an effect is significant.

\subsection{Atmospheric Noise Amplitude}

After showing that our data are consistent with the $\mathrm{K}-\mathrm{T}$ model, we repeated the analysis of Section 3.2 for all of our data. For each observation, we generated 50 simulated atmospheric noise maps with the value of $b$ fixed at $11 / 3$ and the value of $h$ fixed at $1000 \mathrm{~m}$. We set $b=11 / 3$ because this is the power law predicted by the theory and is consistent with our data. The value of $h$ was chosen based on independent measurements of the water vapor profile above Mauna Kea (e.g., Pardo et al. (2001b), estimated from Hilo radiosonde data). Note that our primary result, a measurement of the distribution of $B_{v}^{2}$, does not depend strongly on the choice of $h$ because the best-fit value of $B_{v}^{2}$ is fairly insensitive to $h \cdot{ }^{13}$ For the $143 \mathrm{GHz}$ data, the quartile values of $B_{v}^{2}$ are 100, 280, and $980 \mathrm{mK}^{2} \mathrm{rad}^{-5 / 3}$, and

\footnotetext{
${ }^{13}$ Varying $h$ over the physically reasonable range that we allowed in Section 3.2 (375-6000 m) causes $B_{v}^{2}$ to vary by $\pm 15 \%$ compared to the value of $B_{v}^{2}$ at $h=1000 \mathrm{~m}$. This variation is comparable to the uncertainty in $B_{v}^{2}$ due
} to our flux calibration uncertainty. 

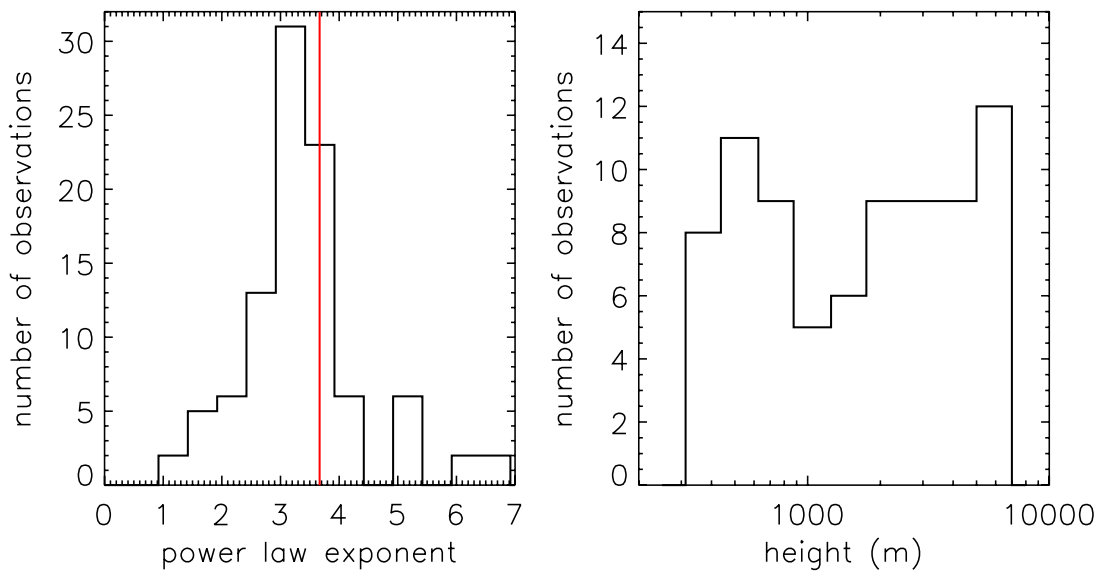

Figure 6. Histogram on the left shows the best-fit value of the power-law exponent $b$ for the K-T model of the atmosphere for a randomly selected subset of 96 $143 \mathrm{GHz}$ observations. The mean is 3.3 and the standard deviation is 1.1 , indicating that our data are consistent with the $\mathrm{K}-\mathrm{T}$ model prediction of $b=11 / 3$, which is shown as a red vertical line. The histogram on the right shows the best-fit value for the height of the turbulent layer. The uniform distribution of $h$ over the allowed range indicates that we do not meaningfully constrain $h$.

(A color version of this figure is available in the online journal.)

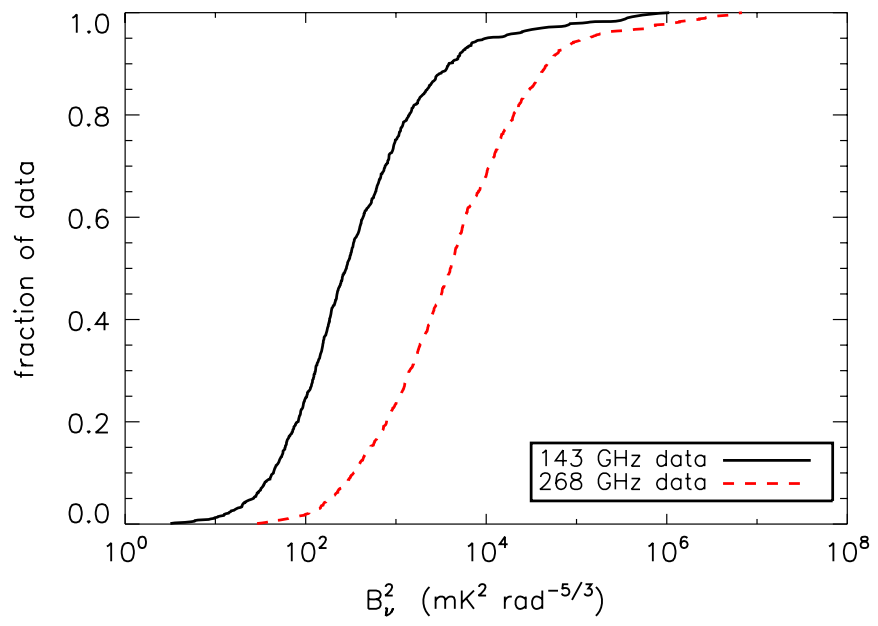

Figure 7. Plots of the cumulative distribution function of $B_{v}^{2}$ at both 143 and $268 \mathrm{GHz}$.

(A color version of this figure is available in the online journal.)

for the $268 \mathrm{GHz}$ data the quartile values are 1100, 4000, and $14000 \mathrm{mK}^{2} \mathrm{rad}^{-5 / 3}$. Note that the uncertainty in these values due to our flux calibration is approximately $12 \%$. Plots of the cumulative distribution function of $B_{v}^{2}$ at each frequency are given in Figure 7.

A reasonable phenomenological expectation is that the fractional fluctuations in the column depth of water vapor are independent of the amount of water vapor (i.e., $\delta \mathcal{C}_{P W} \propto \mathcal{C}_{P W}$ ). Since

$$
B_{v}^{2} \propto\left(\delta \epsilon_{\tau}\right)^{2} \mathrm{~B}_{\mathrm{atm}}^{2},
$$

where $\epsilon_{\tau}=1-e^{\tau_{v}}$ is the emissivity of the atmosphere and $\mathrm{B}_{\mathrm{atm}}=\frac{2 v^{2}}{c^{2}} k_{B} T_{\mathrm{atm}}$ is the brightness of the atmosphere in the Rayleigh-Jeans limit, this means that

$$
B_{v}^{2} \propto\left(\frac{d \epsilon_{\tau}}{d \mathcal{C}_{P W}} \delta \mathcal{C}_{P W}\right)^{2} \mathrm{~B}_{\text {atm }}^{2} \propto\left(\frac{d \epsilon_{\tau}}{d \mathcal{C}_{P W}} \mathcal{C}_{P W}\right)^{2} \mathrm{~B}_{\mathrm{atm}}^{2} .
$$

Note that $\tau_{v}$ is the total opacity of the atmosphere at observing frequency $v$. To test the validity of this expectation, we first considered the data in each observing band separately. The data sets for each observing band spanned a wide range of weather conditions, and in general our predicted scaling fit the data fairly well over the entire range ${ }^{14}$ (see Figure 8). Additionally, we can test our assumption that $\delta \mathcal{C}_{P W} \propto \mathcal{C}_{P W}$ by comparing the values of $B_{v}^{2}$ at $143 \mathrm{GHz}$ to the values at $268 \mathrm{GHz}$. For our bands, the median value of $\left(\frac{d \epsilon_{\tau}}{d \mathcal{C}_{P W}} \mathcal{C}_{P W}\right)^{2} \mathrm{~B}_{\mathrm{atm}}^{2}$, based on the Pardo ATM model (Pardo et al. 2001a, 2001b, 2005), is approximately 16 times larger for the $268 \mathrm{GHz}$ data compared to the $143 \mathrm{GHz}$ data. The ratio of the values of $B_{v}^{2}$ for the two frequencies is 11,14 , and 14 for the three quartiles, indicating that most of the observed difference in $B_{v}^{2}$ between the two observing bands can be accounted for by assuming that $\delta \mathcal{C}_{P W} \propto \mathcal{C}_{P W}$.

\subsection{Comparing Mauna Kea to the South Pole and Atacama}

At the South Pole, the median column depth of precipitable water vapor is $\simeq 0.25 \mathrm{~mm}$, roughly 6-7 times lower than the median value at Mauna Kea. Therefore, the amplitude of the atmospheric noise at the South Pole is expected to be much lower than the amplitude at Mauna Kea. Using our data, along with ACBAR data collected at the South Pole, we can make a direct comparison of the amplitude of the atmospheric noise between the two locations. ACBAR had observing bands centered at 151 and $282 \mathrm{GHz}$, very close to the Bolocam bands, along with a third band centered at $222 \mathrm{GHz}$. For the 2002 observing season, Bussmann et al. (2005) determined that the quartile values of $B_{v}^{2}$ for the $151 \mathrm{GHz}$ band are $3.7,10$, and $37 \mathrm{mK}^{2} \mathrm{rad}^{-5 / 3}$, and the quartile values of $B_{v}^{2}$ for the $282 \mathrm{GHz}$ band are 28,74 , and $230 \mathrm{mK}^{2} \mathrm{rad}^{-5 / 3}$. Therefore, the amplitude of the atmospheric noise is a factor of $\simeq 25$ different for the Bolocam and ACBAR bands at $\simeq 150 \mathrm{GHz}$, and a factor of $\simeq 50$ different for the bands at $\simeq 275 \mathrm{GHz}$. Additionally, the ratio of $B_{v}^{2}$ between Bolocam and ACBAR is similar for all three quartiles in both observing bands, indicating that the relative variations in $B_{v}^{2}$ are comparable at both locations (see Table 1).

Our phenomenological expectation of constant fractional fluctuations in $\mathcal{C}_{P W}$ (i.e., $\frac{\delta \mathcal{C}_{P W}}{\mathcal{C}_{P W}}$ is on average the same at both

\footnotetext{
${ }^{14}$ During the course of our observations $0.5 \lesssim \mathcal{C}_{P W} \lesssim 3.5 \mathrm{~mm}$, and the value of $\left(\frac{d \epsilon_{\tau}}{d \mathcal{C}_{P W}} \mathcal{C}_{P W}\right)^{2} \mathrm{~B}_{\text {atm }}^{2}$ varies by almost 2 orders of magnitude over this range of $\mathcal{C}_{P W}$.
} 

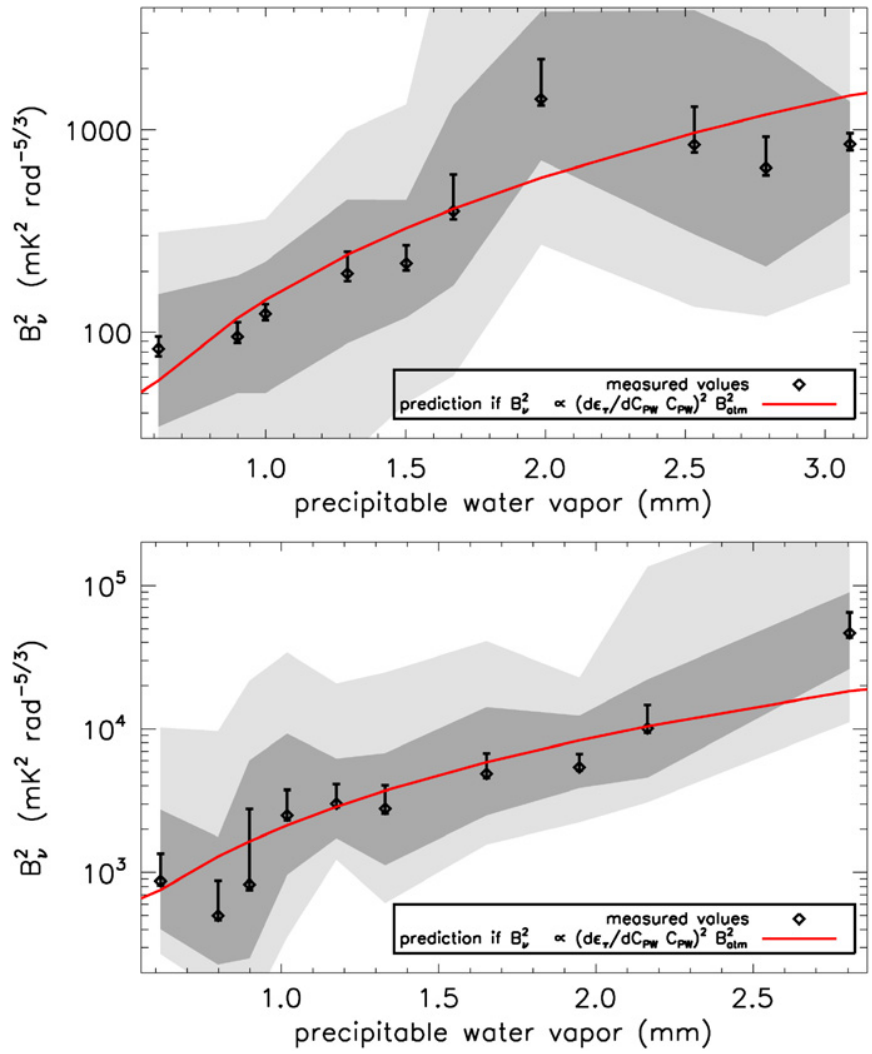

Figure 8. Plots of the amplitude of the atmospheric noise, $B_{v}^{2}$, as a function of column depth of precipitable water vapor, $\mathcal{C}_{P W}$. The data points show the median value of $B_{v}^{2}$ and the error bars give the uncertainty on this median value; the light shaded region spans the 10th-90th centile values of $B_{v}^{2}$, and the darker shaded region spans the 25th-75th centile values of $B_{v}^{2}$. The top plot shows Bolocam data collected at $143 \mathrm{GHz}$ and the bottom plot shows Bolocam data collected at $268 \mathrm{GHz}$. Overlaid on the plots is a fit to the data assuming that the fractional fluctuations in the column depth of precipitable water are constant (i.e., that $B_{v}^{2}$ is proportional to $\left(\frac{d \epsilon_{\tau}}{d \mathcal{C}_{P W}} \mathcal{C}_{P W}\right)^{2} \mathrm{~B}_{\text {atm }}^{2}$ ).

(A color version of this figure is available in the online journal.)

locations) implies that the ratio of $\left(\frac{d \epsilon_{\tau}}{d \mathcal{C}_{P W}} \mathcal{C}_{P W}\right)^{2} \mathrm{~B}_{\text {atm }}^{2}$ should predict the ratio of $B_{v}^{2}$. This prediction, again based on the Pardo ATM model (Pardo et al. 2001a, 2001b, 2005) ${ }^{15}$ is that the ratio of $B_{v}^{2}$ should be 12 for the $\simeq 150 \mathrm{GHz}$ bands and 21 for the

\footnotetext{
${ }^{15}$ Note that by adjusting the input parameters, the Pardo ATM model can be
} matched to the conditions at the South Pole. $\simeq 275 \mathrm{GHz}$ bands. ${ }^{16}$ These predicted scalings are much lower than the observed scalings of 25 and 50, indicating that the value of $\left(\frac{\delta \mathcal{C}_{P W}}{\mathcal{C}_{P W}}\right)^{2}$ is a factor of $\simeq 2$ lower at the South Pole compared to Mauna Kea. Consequently, in addition to the South Pole being on average much drier than Mauna Kea, we conclude that the fractional fluctuations in the column depth of water vapor are also lower by a factor of $\simeq \sqrt{2}$.

Thus, because $\left(\frac{\delta \mathcal{C}_{P W}}{\mathcal{C}_{P W}}\right)^{2}$ is a factor of $\simeq 2$ larger at Mauna Kea compared to the South Pole, and because the median value of $\mathcal{C}_{P W}^{2}$ is a factor of $\simeq 40$ larger at Mauna Kea compared to the South Pole, we find that $B_{v}^{2}$ is a factor of $\simeq 80$ larger at Mauna Kea compared to the South Pole for millimeter-wave observations. Additionally, Bussmann et al. (2005), using the results in Lay \& Halverson (2000), found that the value of $B_{v}^{2}$ is a factor of $\simeq 30$ lower at the South Pole compared to the ALMA site in Atacama. Therefore, we can infer that $B_{v}^{2}$ is a factor of $\simeq 3$ lower at the ALMA site compared to Mauna Kea. Since the value of $\left(\frac{d \epsilon_{\tau}}{d \mathcal{C}_{P W}} \mathcal{C}_{P W}\right)^{2} \mathrm{~B}_{\text {atm }}^{2}$ is a factor of $\simeq 3.5$ lower at the ALMA site than Mauna Kea for the median observing conditions at each location, we find that the value of $\left(\frac{\delta \mathcal{C}_{P W}}{\mathcal{C}_{P W}}\right)^{2}$ is similar for Mauna Kea and the ALMA site. ${ }^{17}$ Therefore, the fractional fluctuations in the column depth of precipitable water vapor appear to be the same at Mauna Kea and the ALMA site, but they are significantly lower at the South Pole; these lower fluctuations at the South Pole may be due to the lack of diurnal variations at that site. We emphasize that these are statements about the fluctuations in $\mathcal{C}_{P W}$, and thus relate only to atmospheric noise. In shorter wavelength bands with higher opacity, it may be that signal attenuation and photon noise due to the absolute opacity are more important than atmospheric noise in determining the quality of a given site.

\footnotetext{
16 We have used the measured Bolocam and ACBAR bandpasses, along with the Pardo ATM model (Pardo et al. 2001a, 2001b, 2005), to determine the value of $\left(\frac{d \epsilon_{\tau}}{d \mathcal{C}_{P W}} \mathcal{C}_{P W}\right)^{2} \mathrm{~B}_{\text {atm }}^{2}$ for each instrument for the median observing conditions at their respective sites. Although the Bolocam and ACBAR bands are similar, there are important differences; not only are the Bolocam bands centered at lower frequencies than the ACBAR bands, but the $\simeq 150 \mathrm{GHz}$ Bolocam band is significantly narrower as well. Since the value of $\left(\frac{d \epsilon_{\tau}}{d \mathcal{C}_{P W}}\right)^{2}$ is, in general, a strong function of observing frequency, these subtle differences in the observing bands produce noticeable differences in the predicted value of $B_{v}^{2}$. Additionally, differences in the atmosphere above each location can cause significant differences in the value of $\left(\frac{d \epsilon_{\tau}}{d \mathcal{C}_{P W}}\right)^{2}$ for a given value of $\mathcal{C}_{P W}$. Specifically, the ratio of $\left(\frac{d \epsilon_{\tau}}{d \mathcal{C}_{P W}}\right)^{2}$ between Bolocam and ACBAR is $\simeq 0.30$ for the $\simeq 150 \mathrm{GHz}$ bands and $\simeq 0.45$ for the $\simeq 275 \mathrm{GHz}$ bands.

17 The median value of $\mathcal{C}_{P W}$ at the Cerro Chajnantor site under consideration for the Cornell-Caltech Atacama Telescope (CCAT) is approximately $0.83 \mathrm{~mm}$, so the median value of $B_{v}^{2}$ should be about $30 \%$ lower at the CCAT
} site compared to the ALMA site.

Table 1

Atmospheric Noise Amplitude $\left(B_{v}^{2}\right)$

\begin{tabular}{lcccc}
\hline \hline Instrument & Frequency & Quartile 1 & Quartile 2 & Quartile 3 \\
\hline Bolocam & $143 \mathrm{GHz}$ & $100 \mathrm{mK}^{2} \mathrm{rad}^{-5 / 3}$ & $280 \mathrm{mK}^{2} \mathrm{rad}^{-5 / 3}$ & $980 \mathrm{mK}^{2} \mathrm{rad}^{-5 / 3}$ \\
ACBAR & $151 \mathrm{GHz}$ & $3.7 \mathrm{mK}^{2} \mathrm{rad}^{-5 / 3}$ & $10 \mathrm{mK}^{2} \mathrm{rad}^{-5 / 3}$ & $37 \mathrm{mK}^{2} \mathrm{rad}^{-5 / 3}$ \\
Bolocam/ACBAR & & 27 & 28 & 26 \\
\hline Bolocam & $268 \mathrm{GHz}$ & $1100 \mathrm{mK}^{2} \mathrm{rad}^{-5 / 3}$ & $4000 \mathrm{mK}^{2} \mathrm{rad}^{-5 / 3}$ & $14000 \mathrm{mK}^{2} \mathrm{rad}^{-5 / 3}$ \\
ACBAR & $282 \mathrm{GHz}$ & $28 \mathrm{mK}^{2} \mathrm{rad}^{-5 / 3}$ & $74 \mathrm{mK}^{2} \mathrm{rad}^{-5 / 3}$ & $230 \mathrm{mK}^{2} \mathrm{rad}^{-5 / 3}$ \\
Bolocam$/$ ACBAR & & 39 & 54 & 61
\end{tabular}

Notes. The observed quartile values of $B_{v}^{2}$ for the two Bolocam observing bands from Mauna Kea and two of the ACBAR observing bands from the South Pole. The ratio of $B_{v}^{2}$ for the two instruments is given for each of the bands $(\simeq 150$ and $\simeq 275 \mathrm{GHz})$. 


\subsection{Map Variance as a Function of Atmospheric Conditions}

Although it is useful to determine the amplitude of the fluctuations in atmospheric emission, the quality of our data is characterized by the residual noise level after removing as much atmospheric noise as possible. We will use the difference between the measured map variance, $\sigma_{\text {map }}^{2}$, and the expected map variance in the absence of atmospheric noise, $\sigma_{\text {white }}^{2}$, as a proxy for this residual noise level. Note that these maps are produced after removing most of the atmospheric noise using the average subtraction algorithm given in Section 4.1, and $\sigma_{\text {white }}^{2}$ is estimated from the noise level of the map at high spatial frequency where the atmospheric noise is negligible.

As expected, we find a correlation between $\sigma_{\text {map }}^{2}-\sigma_{\text {white }}^{2}$ and $B_{v}^{2}$, although there is quite a bit of scatter in the amount of residual atmospheric noise for a given value of $B_{v}^{2}$ (see Figure 9). Most of this scatter is likely due to the fact that the residual noise is inversely proportional to the amount of correlation in the atmospheric signal over our FOV; this correlation depends not only on the value of $B_{v}^{2}$, but also on the height and angular wind speed of the turbulent layer. Since the atmosphere is in the near field for Bolocam, an increase in the height of the turbulent layer reduces the overlap of the beams from individual detectors. Thought of in a different way, a decrease in the height of the turbulent layer implies that the beam smoothing of the atmospheric signal is extended to larger spatial scales, making the atmospheric signal more uniform over the fixed angular scale of our FOV. Therefore, for a fixed value of $B_{v}^{2}$, there will be less correlation in the atmospheric signal over the FOV as the height of the turbulent layer increases. Additionally, the angular wind speed of the turbulent layer will influence the amount of atmospheric noise in the data because our scan speed is much slower than the angular wind speed. This means that a higher angular wind speed will modulate the atmospheric noise to higher frequencies in the time-stream data; at higher frequencies more of the atmospheric noise will be in our signal band and less of the noise will be removed using the subtraction algorithms described in Section 4. Also, note that, in the best conditions, our data approach the white noise limit, and these conditions can occur over a relatively wide range of values for $B_{v}^{2}$. Thus, we find that while $B_{v}^{2}$ (and also $\mathcal{C}_{P W}$ based on our assumption that $\left.B_{v}^{2} \propto\left(\frac{d \epsilon_{\tau}}{d \mathcal{C}_{P W}} \mathcal{C}_{P W}\right)^{2} \mathrm{~B}_{\text {atm }}^{2}\right)$ is not a precise predictor of $\sigma_{\text {map }}^{2}-\sigma_{\text {white }}^{2}$, there is a general trend of less residual map noise at lower values of $B_{v}^{2}\left(\mathcal{C}_{P W}\right)$.

\subsection{Summary}

In summary, the $\mathrm{K}-\mathrm{T}$ thin-screen model appears to provide an adequate description of the atmospheric signal in our data. We find the angular speed of the thin screen to be approximately $30 \operatorname{arcmin~s}^{-1}$, although roughly half of our data contain some features that cannot be explained with a single angular wind velocity. The turbulent layer has a power-law exponent of $b=3.3 \pm 1.1$, consistent with the K-T prediction of $b=11 / 3$. If we assume that $b=11 / 3$, then the median amplitude of the atmospheric fluctuations is $280 \mathrm{mK}^{2} \mathrm{rad}^{-5 / 3}$ at $143 \mathrm{GHz}$ and $4000 \mathrm{mK}^{2} \mathrm{rad}^{-5 / 3}$ at $268 \mathrm{GHz}$. These amplitudes are $\simeq 80$ times larger than the amplitudes found at similar observing frequencies at the South Pole using ACBAR (Bussmann et al. 2005). Most of the scaling in $B_{v}^{2}$ between observing frequencies and locations can be accounted for by assuming that the fractional fluctuations in the column depth of precipitable
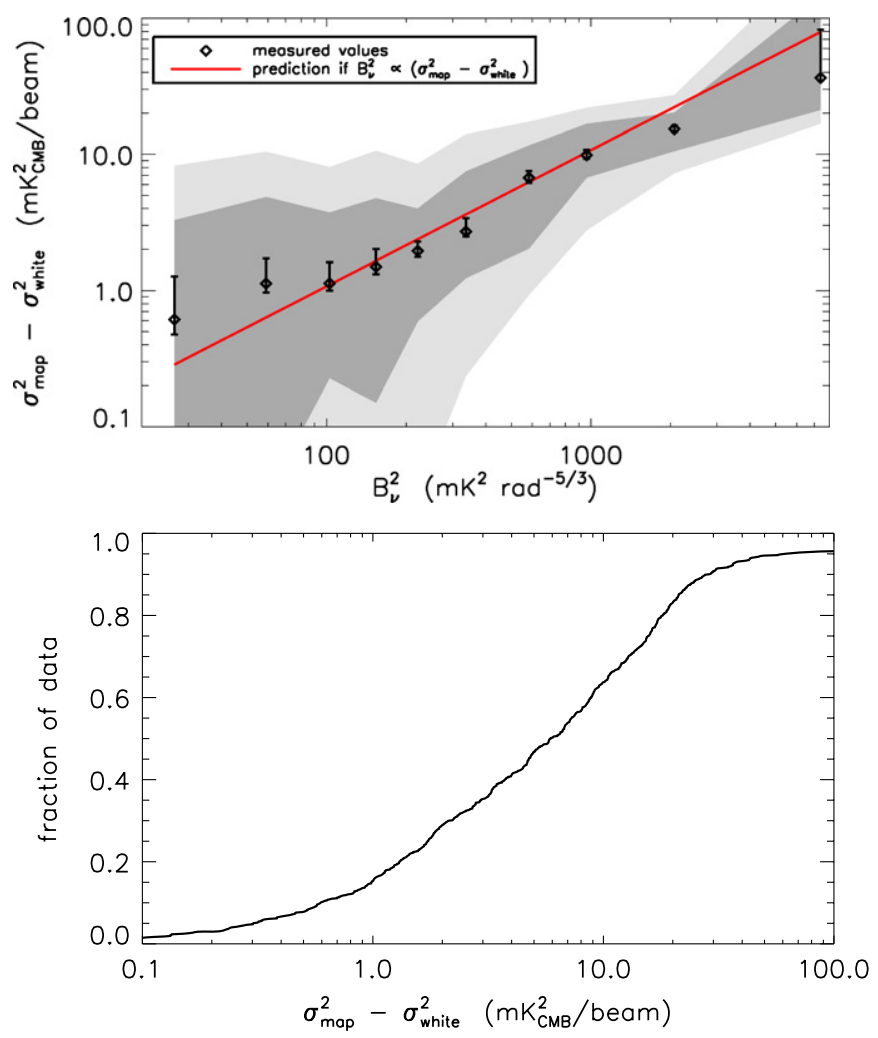

Figure 9. Top plot shows the $143 \mathrm{GHz}$ single-observation residual map variance after subtracting the white noise level as a function of the amplitude of the atmospheric noise, $B_{v}^{2}$. Note that the typical white noise level of the maps is $\sigma_{\text {white }}^{2} \simeq 5 \mathrm{mK}_{\mathrm{CMB}}^{2}$. The error bars represent the error on the median value for each data point; the light shaded region spans the 10th-90th centile values and the dark shaded region spans the 25th-75th centile values. Note that most of the atmospheric noise has been removed from the data using the average subtraction algorithm described in Section 4.1. The red line shows the prediction assuming that the residual map variance is proportional to $B_{v}^{2}$. The bottom plot shows cumulative distribution of residual map variance for the $143 \mathrm{GHz}$ data.

(A color version of this figure is available in the online journal.)

water vapor, $\frac{\delta \mathcal{C}_{P W}}{\mathcal{C}_{P W}}$, are constant. However, the data indicate that $\frac{\delta \mathcal{C}_{P W}}{\mathcal{C}_{P W}}$ is a factor of $\simeq \sqrt{2}$ smaller at the South Pole compared to Mauna Kea. We thus find that the bulk of the reduction in atmospheric noise at the South Pole is due to the consistently low value of $\mathcal{C}_{P W}$ at that site, and the lower fractional fluctuations in the precipitable water vapor only reduce the rms of the atmospheric noise by an additional factor of $\simeq \sqrt{2}$. Additionally, after removing as much atmospheric noise as possible, we find a correlation between the value of $B_{v}^{2}$ and the amount of residual atmospheric noise in our data, although it is likely that the height and angular speed of the turbulent layer also influence the amount of residual atmospheric noise.

\section{ATMOSPHERIC NOISE: REMOVAL}

In this section, we describe various atmospheric noise removal techniques, including one based on the relatively unsophisticated common-mode assumption and several based on the properties of the atmospheric noise determined from our fits to the K-T model. Additionally, we summarize the results of subtracting the atmospheric noise using adaptive principle component analysis (PCA). Note that in this section, along with Section 5, our analysis focuses entirely on the $143 \mathrm{GHz}$ data. 

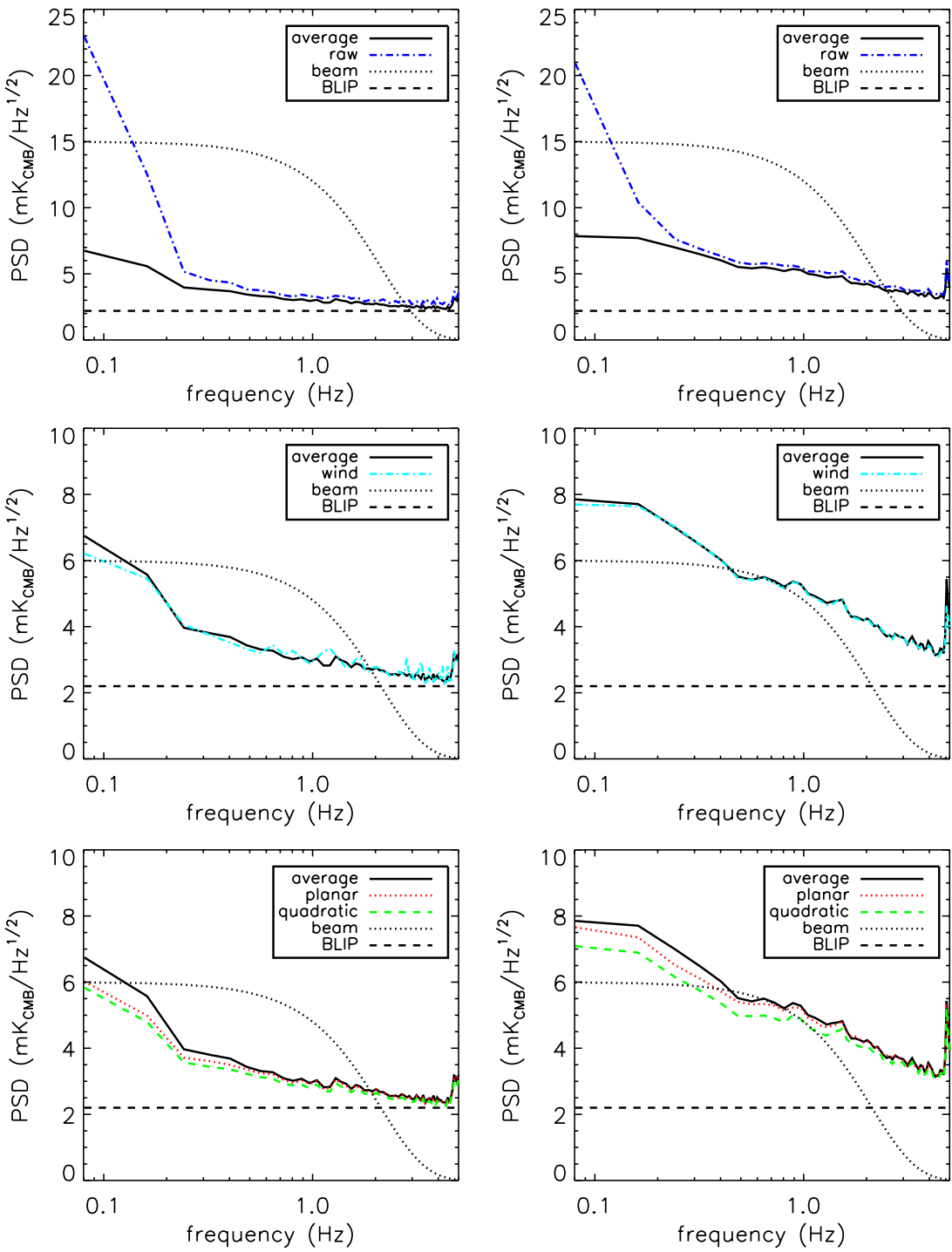

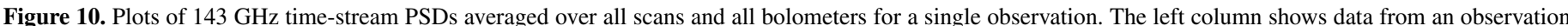

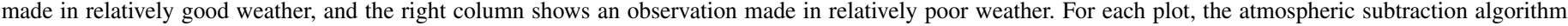

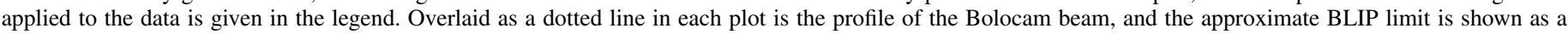
dashed line.

(A color version of this figure is available in the online journal.)

\subsection{Average Template Subtraction}

Our most basic method for removing atmospheric noise is to subtract the signal that is common to all of the bolometers. Initially, a template is constructed according to

$$
T_{n}=\frac{\sum_{i=1}^{i=N_{b}} c_{i}^{-1} d_{i n}}{\sum_{i=1}^{i=N_{b}} c_{i}^{-1}}
$$

where $n$ is the sample number, $N_{b}$ is the number of bolometers, $c_{i}$ is the relative responsivity of bolometer $i, d_{i n}$ is the signal recorded by bolometer $i$ at sample number $n$, and $T_{n}$ is the template. The relative responsivity is required to account for the fact that the bolometer response (in $\mathrm{nV}$ ) to a given signal (in $\mathrm{mK}$ ) is slightly different from one bolometer to the next. A separate template is computed for each $\simeq 15$-s-long scan. After the template is computed, it is correlated with the signal from each bolometer to determine the correlation coefficient, with

$$
\tilde{c_{i}}=\frac{\sum_{j=1}^{j=N_{s}} T_{n} d_{i n}}{\sum_{j=1}^{j=N_{s}} T_{n}^{2}} .
$$

$\tilde{c_{i}}$ is the correlation coefficient of bolometer $i$, and $N_{s}$ is the number of samples in the $\simeq 15$-s-long scan. ${ }^{18}$ Next, the $c_{i}$ in Equation (1) are set equal to the values of $\tilde{c}_{i}$ found from Equation (1), and a new template is computed. The process is repeated until the values of $c_{i}$ stabilize. We generally iterate until the average fractional change in the $c_{i}$ s is less than $1 \times 10^{-8}$, which takes five to ten iterations. If the $c_{i}$ s fail to converge after 100 iterations, then the scan is discarded from the data. This algorithm generally removes the majority of the atmospheric noise, as shown in Figure 10.

\footnotetext{
18 The best-fit correlation coefficients change from one scan to the next, typically by a couple percent.
} 


\subsection{Wind Model}

Since the moving screen atmospheric model given in Section 3.1 provided a fairly good description of our data, we attempted to improve our atmospheric noise removal algorithm by applying the appropriate time delay/advance to every bolometer prior to average subtraction. The angular wind velocity for each observation was determined using the formalism described in Section 3.1, and from this angular wind velocity we computed the time delay/advance for each bolometer based on its location on the focal plane. If the spatial structure of the atmospheric emission is static on the timescales of the delay/advance, then the shifted beam centers will be pointed at the same location in the turbulent layer for bolometers aligned parallel to the angular wind velocity. Therefore, the atmospheric signal in these shifted time-streams will be identical for these bolometers, modulo uncertainties in the angular wind velocity, slight differences in the beam profiles, etc. See Section 3.2 for a discussion of the impact of the latter. For the typical angular speeds of the turbulent layer, the shifts are of order 1 sample, and we used a linear interpolation to account for shifts that are a fraction of a sample. Note that this linear interpolation acts as a low-pass filter on our data; to preserve the PSDs of our time-streams, we correct for this attenuation in frequency space (see the appendix). We applied the appropriate shift to the time-stream of each bolometer before performing average subtraction, but this did not seem to reduce the post-subtraction noise PSD relative to time-instantaneous average subtraction (see Figure 10). Therefore, we abandoned this atmospheric noise subtraction algorithm.

\subsection{Higher Order Template Subtraction}

Based on the K-T model fits, we were able to determine which spatial Fourier modes cause the atmospheric emission to become uncorrelated over our $8^{\prime}$ FOV. Our time-stream PSDs show that most of the atmospheric noise signal is at frequencies below $0.1 \mathrm{~Hz}$, and the atmospheric noise becomes negligible at frequencies above $0.5 \mathrm{~Hz}$. Therefore, most of the atmospheric fluctuations occur on long timescales, which correspond to large spatial scales. To convert these temporal frequencies to angular frequencies, we divide by the angular wind speed we determined for the thin-screen model, which we found in Section 3.1 to be approximately $30 \operatorname{arcmin~} \mathrm{s}^{-1}$. This means that most of the atmospheric noise is at small angular frequencies with $\alpha<300^{-1} \operatorname{arcmin}^{-1}$, and the atmospheric noise is negligible for angular frequencies larger than $\alpha=60^{-1} \mathrm{arcmin}^{-1}$. We can therefore conclude that very little atmospheric signal is sourced by spatial modes with wavelengths smaller than our FOV. Note that Jenness et al. (1998), based on the atmospheric noise in SCUBA data and making reasonable assumptions for the height and angular speed of the turbulent layer, found a similar scale for the atmospheric fluctuations.

Since most of the atmospheric signal is caused by power in spatial modes with wavelengths much larger than our FOV, the signal will be slowly varying over our focal plane. Therefore, we decided to model the atmospheric fluctuations using a low-order two-dimensional polynomial in detector position. This is similar to the method used by SHARC II to remove atmospheric noise (Kovacs 2008). Additionally, Borys et al. (1999) attempted a similar planar subtraction with SCUBA, although with limited success.

For planar and quadratic subtraction, including the special case of average subtraction described in Section 4.1, the algorithm is implemented as follows. The data are modeled accord- ing to

$$
\vec{d}_{n}=\mathbf{C S} \vec{p}_{n},
$$

where $\vec{d}_{n}$ is a vector with $n_{b}$ elements representing the bolometer data at time sample $n, \mathbf{C}$ is a diagonal $n_{b} \times n_{b}$ element matrix with the relative responsivity of each bolometer, $\mathbf{S}$ is an $n_{b} \times n_{\text {params }}$ element matrix, and $\vec{p}_{n}$ is a vector with $n_{\text {params }}$ elements. $n_{b}$ is the number of bolometers, $n$ is the sample number within the $\simeq 15$-s-long scan, and $n_{\text {params }}$ is the number of fit parameters. $\mathbf{S}$ is based on the geometry of the focal plane, with $n_{\text {params }}=1 / 3 / 6$ for average/planar/quadratic subtraction and

$$
\begin{array}{rll}
\mathbf{S}_{i 1}=1, & \mathbf{S}_{i 2}=x_{i}, & \mathbf{S}_{i 3}=y_{i} \\
\mathbf{S}_{i 4}=x_{i} y_{i}, & \mathbf{S}_{i 5}=x_{i}^{2}, & \mathbf{S}_{i 6}=y_{i}^{2},
\end{array}
$$

where $\vec{x}$ and $\vec{y}$ are vectors with $n_{b}$ elements that contain the $x$ and $y$ coordinate of each bolometer on the focal plane. The $\vec{p}_{n}$ are the $n_{\text {params }}$ atmospheric noise templates, which are obtained by minimizing

$$
\chi_{n}^{2}=\left(\vec{d}_{n}-\mathbf{C S} \vec{p}_{n}\right)^{T}\left(\vec{d}_{n}-\mathbf{C S} \vec{p}_{n}\right)
$$

with respect to $\vec{p}_{n}{ }^{19}$ For a given time sample $n$, the values of $\vec{p}_{n}$ give the coefficients for each term in the polynomial expansion of the atmospheric signal over the focal plane at that particular time. A single element in the vector $\vec{p}_{n}$, when considered over all the samples in a scan, gives the time dependence of that particular coefficient. Essentially, each element in $\vec{p}_{n}$ can be thought of as a data time-stream that gives the amplitude of the atmospheric signal with a particular spatial dependence over the focal plane. Minimizing Equation (1) yields

$$
\vec{p}_{n}=\left(\mathbf{S}^{T} \mathbf{S}\right)^{-1} \mathbf{S}^{T} \mathbf{C}^{-1} \vec{d}_{n} .
$$

Once $\vec{p}_{n}$ is known, we can construct an atmospheric template analogous to Equation (1) for each bolometer according to

$$
\vec{T}_{n}=\mathbf{S} \vec{p}_{n} .
$$

Note that $\vec{T}_{n}$ varies from bolometer to bolometer as prescribed by the assumed two-dimensional polynomial form and the bestfit polynomial coefficients $\vec{p}_{n}$. A correlation coefficient is then computed for each bolometer according to Equation (1), a new matrix $\mathbf{C}$ is computed according to these correlation coefficients, and a new template is computed according to Equations (6) and (7). The process is repeated until the fractional change in the values of the correlation coefficients is less than one part in $10^{8}$.

In general, the PSDs of the higher order templates are $\simeq 5$ times smaller than the PSD of the zeroth-order template for bolometers halfway between the center and the edge of the focal plane. As expected, the ratio of the higher order templates to the

\footnotetext{
19 We have assumed that the individual bolometer intrinsic (i.e., non-atmospheric) noises at time sample $n$ are not correlated with each other so that the covariance matrix is diagonal. The noises of the different bolometers are sufficiently similar, once corrected for relative responsivity via $\mathbf{C}$, that the noise covariance matrix can, in fact, be taken to be a multiple of the identity matrix. The $\chi^{2}$ statistic is thus proportional to a statistically rigorous $\chi^{2}$, though it is not normalized correctly. The normalization is unimportant for our purposes. If these assumptions are incorrect, then our estimators of the atmospheric templates will not be minimum variance estimators; they will, however, be unbiased. We also have implicitly assumed that we should determine $\vec{p}_{n}$ at each point in time independently, which relies on the assumption that the intrinsic noise of a given bolometer is uncorrelated with itself in time (i.e., white in frequency space). This is also a reasonably valid assumption, and, again, if it is incorrect, then our estimators are not maximally
} efficient but remain unbiased. 

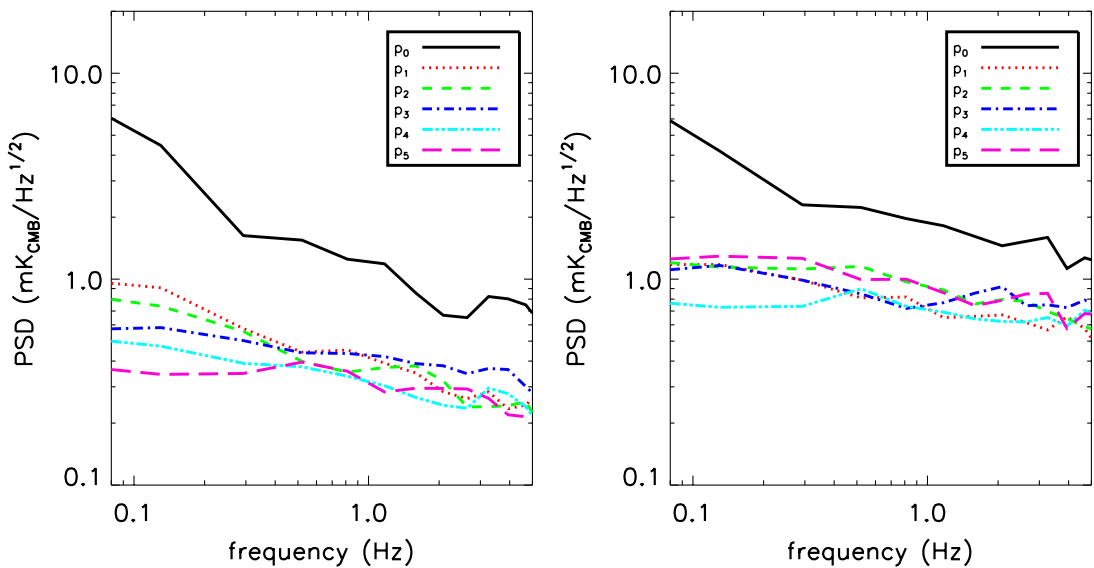

Figure 11. Power spectra for the templates generated by the quadratic sky subtraction algorithm for $143 \mathrm{GHz}$ data. The plot on the left represents data collected in relatively good weather, and the plot on the right shows data collected in relatively poor weather. All six elements of $\vec{p}_{i}$ are plotted, with labels given in the upper right of each plot. The higher order elements in $\vec{p}_{i}$ are shown for a bolometer approximately halfway between the array center and the edge of the array. Note that the magnitude of the higher order templates in bad weather is a factor of $\simeq 2$ larger than the magnitude of the higher order templates in good weather.

(A color version of this figure is available in the online journal.)

zeroth-order template increases as the weather becomes worse. Some typical power spectra of the $\vec{p}_{i}$ are shown in Figure 11.

Compared to average sky subtraction, a slight reduction in noise, most noticeable at low frequencies, can be seen in the time-streams (see Figure 10). However, the difference in the noise level of a map made from co-adding all $\simeq 500$ observations of the Lynx science field is far more dramatic (see Figure 12). The reason such a small change in the time-stream PSDs produces such a large change in the map PSDs is because planar and quadratic subtraction reduce the amount of residual atmospheric noise correlations remaining in the time-streams of the bolometers. Figures 13 and 14 illustrate this reduction in the bolometer-bolometer correlations with quadratic subtraction.

However, the higher order templates also remove more astronomical signal compared to average subtraction. Therefore, a single observation of a given astronomical source shape will have an optimal subtraction algorithm based on the noise level of the data and the amount of signal attenuation. For an extended source, (e.g., a CMB anisotropy, which is usually modeled as flat in $C_{\ell} \ell(\ell+1) / 2 \pi$ at large $\ell$, where $\ell$ is angular multipole), ${ }^{20}$ we found that average subtraction was optimal for $\simeq 50 \%$ of the observations, planar subtraction was optimal for $\simeq 42 \%$ of the observations, and quadratic subtraction was optimal for $\simeq 8 \%$ of the observations. Average and planar subtraction provide very similar sensitivity to a flat CMB power spectrum, likely because the CMB signal is nearly indistinguishable from the atmospheric noise signal for linear variations over our $8^{\prime}$ FOV (see Figure 12). For point-like sources, we found that average subtraction was optimal for $\simeq 37 \%$ of the observations, planar subtraction was optimal for $\simeq 49 \%$ of the observations, and quadratic subtraction was optimal for $\simeq 14 \%$ of the observations. Most observations were optimally processed with the same algorithm for both point-like and extended objects, indicating that weather is the primary factor in determining which subtraction algorithm will be optimal for a given observation. However, observations of

\footnotetext{
20 A flat CMB anisotropy signal profile is used throughout this paper to quantify the sensitivity of our data and to test our subtraction algorithms. This signal shape was chosen because: (1) the $143 \mathrm{GHz}$ data were collected primarily to look for CMB anisotropies (Sayers et al. 2009); (2) it has a similar power spectrum to the atmospheric noise, making it a good indicator of the amount of atmospheric noise; and (3) several large-format instruments have also been commissioned at millimeter wavelengths to study the CMB anisotropies at the South Pole (e.g., SPT; Ruhl et al. 2004) and at Atacama (APEX-SZ and ACT-Dobbs et al. 2006; Kosowsky 2003).
}

point sources show a slight preference for planar and quadratic subtraction compared to extended sources. This is because the higher order subtraction algorithms attenuate signal primarily on large scales, so extended objects are more sensitive to the signal loss caused by these algorithms.

\subsection{Adaptive PCA}

We have also used an adaptive PCA algorithm to remove atmospheric noise from Bolocam data (Laurent et al. 2005; Murtagh \& Heck 1987). The motivation for this algorithm is to produce a set of statistically independent modes, which hopefully convert the widespread spatial correlations into a small number of high variance modes. First, consider the meansubtracted bolometer data for a single scan to be a matrix, $\mathbf{d}$, with $n_{b} \times n_{s}$ elements. As usual, $n_{b}$ denotes the number of bolometers and $n_{s}$ denotes the number of samples in a scan. For our adaptive PCA algorithm, we first calculate a covariance matrix, $\mathbf{C}$, with $n_{b} \times n_{b}$ elements according to

$$
\mathbf{C}=\mathbf{d d}^{T} .
$$

Next, $\mathbf{C}$ is diagonalized in the standard way to produce a set of eigenvalues $\left(\lambda_{i}\right)$ and eigenvectors $\left(\vec{\phi}_{i}\right)$, where $i$ is the index of the eigenvector and $\vec{\phi}$ contains $n_{b}$ elements. The $j$ th element of the $i$ th eigenvector, $\left(\phi_{i}\right)_{j}$, indicates the contribution of the $j$ th bolometer to the $i$ th eigenvector. The $i$ th eigenvalue gives the contribution of the $i$ th eigenvector to the total variance of the data. Eigenvectors with large eigenvalues thus carry most of the noise in the time-stream data. A transformation matrix, $\mathbf{R}$, is then formed from the eigenvectors according to

$$
\mathbf{R}=\left(\vec{\phi}_{1}, \vec{\phi}_{2}, \ldots, \overrightarrow{\phi_{n_{b}}}\right) .
$$

This transformation matrix is used to decompose the data into eigenfunctions, $\vec{\Phi}_{i}$, with

$$
\left(\vec{\Phi}_{1}, \vec{\Phi}_{2}, \ldots, \vec{\Phi}_{n_{b}}\right)^{T}=\mathbf{\Phi}=\mathbf{d R}^{T} .
$$

These eigenfunctions are the time-dependent amplitude of the corresponding eigenvector in the time-stream data; the eigenvalue $\lambda_{i}$ is the variance of that time-dependent eigenfunction. At this point, we compute the logarithm for all of the eigenvalues, 

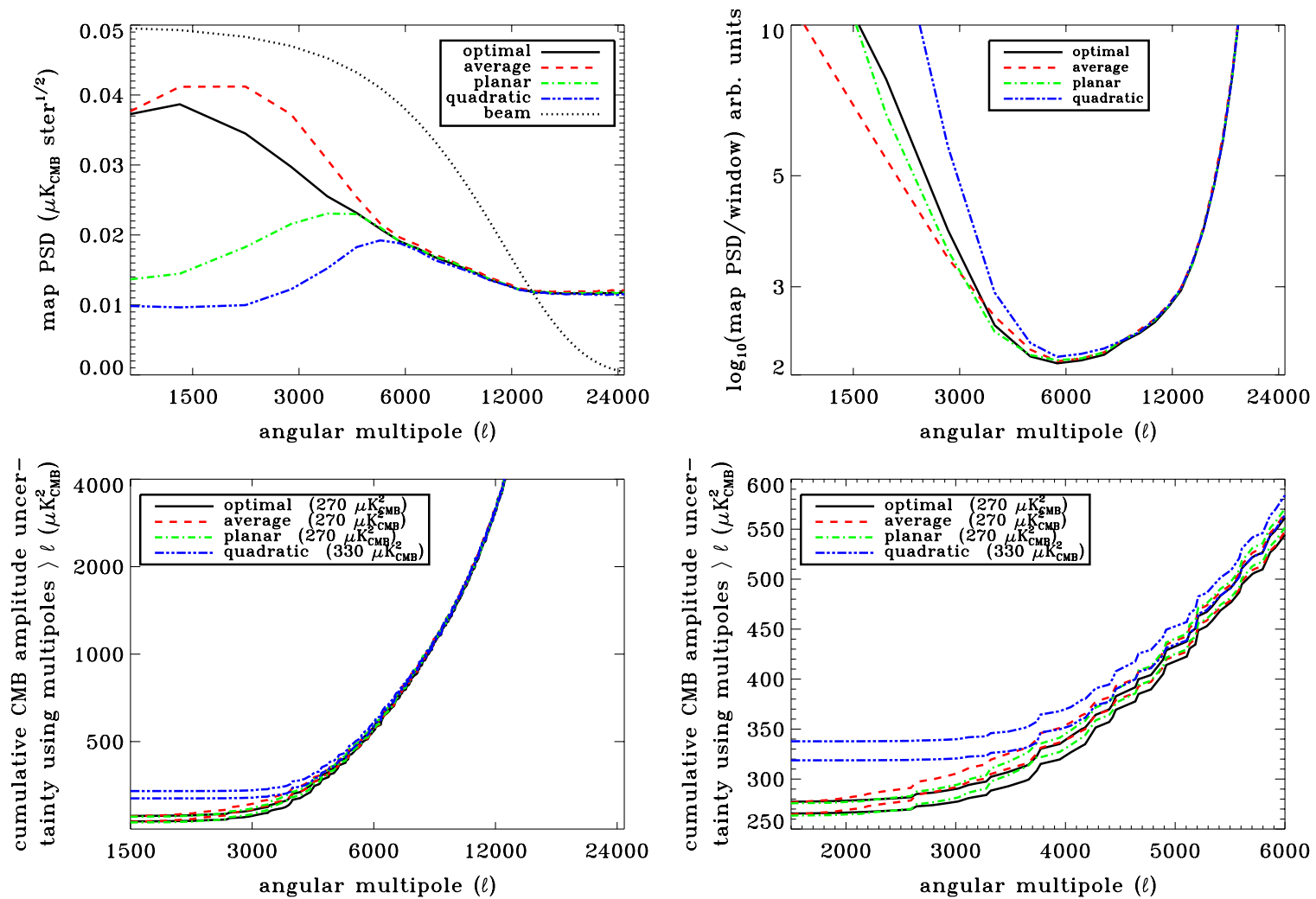

Figure 12. Plot in the top left shows the map PSD for all of the $143 \mathrm{GHz}$ Lynx field data processed using average subtraction, planar subtraction, quadratic subtraction, or the optimal subtraction for each observation. The plot in the top right shows the same data divided by the window function for each subtraction algorithm and the window function of the beam. This plot shows the relative sensitivity per unit $\Delta \log (\ell)$ to a flat band power CMB power spectrum in $C_{\ell} \ell(\ell+1) / 2 \pi$. The bottom plots show the cumulative sensitivity to a flat band power CMB power spectrum including all of the data at multipoles $>\ell$. The two curves for each data set represent the uncertainty based on the rms variations in each $\ell$-bin. Note that the sensitivity, including all $\ell$-bins, is consistent for the average, planar, and optimal data sets. Therefore, our sensitivity to a CMB signal is largely independent of whether average or planar subtraction is used. This result implies that the CMB signal and the atmospheric noise signal are nearly indistinguishable if they are modeled as linearly varying over our $8^{\prime}$ FOV. However, since quadratic subtraction reduces our sensitivity, we can infer that the CMB signal shows more correlation on small scales than the atmospheric noise signal, which is reasonable since the power spectrum of the atmosphere goes like $\alpha^{-11 / 3}$ and the power spectrum of the CMB goes like $\alpha^{-2}$.

(A color version of this figure is available in the online journal.)

and then determine the standard deviation of that distribution. All of the eigenvalues with a logarithm more than three standard deviations from the mean are cut, and then a new standard deviation is calculated. The process is repeated until there are no more outliers with large eigenvalues. Next, all of the eigenvector columns $\vec{\phi}_{i}$ in $\mathbf{R}$ that correspond to the cut eigenvalues are set to zero, yielding a new transformation matrix, $\mathbf{R}^{\prime}$. When reconstructing the data, setting these columns in $\mathbf{R}$ equal to zero is equivalent to discarding the cut eigenvectors. Finally, we transform back to the original basis, with the adaptive PCA cleaned data, $\mathbf{d}^{\prime}$, computed according to

$$
\mathbf{d}^{\prime}=\Phi \mathbf{R}^{\prime}
$$

In general, the eigenfunction, $\vec{\Phi}_{i}$, corresponding to the largest eigenvalue is nearly equal to the template created for average sky subtraction. Therefore, the physical interpretation of the leading order eigenfunction is fairly well understood. However, it is not obvious what signal(s) the lower order eigenfunctions correspond to.

Typically, adaptive PCA only removes one or two eigenvectors from the $143 \mathrm{GHz}$ data. In good weather, adaptive PCA produces slightly better time-stream noise PSDs than average subtraction, while average subtraction produces slightly better noise PSDs in bad weather (see Figure 15). However, adaptive PCA attenuates much more signal than average subtraction at low frequencies, which means that average subtraction produces a better post-subtraction signal-to-noise ratio $(\mathrm{S} / \mathrm{N})$ compared to adaptive PCA subtraction in all conditions. Therefore, adaptive PCA was never the optimal subtraction algorithm for our analysis of blankfield data. Note that for observations of bright sources an iterative map-making technique can be used to recover a substantial amount of the signal that is lost in the process of subtracting the atmospheric noise (Enoch et al. 2006). Such flux recovery may change which subtraction algorithm that is optimal for a given observation.

\subsection{Prospects for Improving Atmospheric Noise Subtraction}

Although none of our subtraction algorithms allow us to reach BLIP-limited performance with Bolocam below $\simeq 0.5 \mathrm{~Hz}$, this does not mean that BLIP performance is impossible from Mauna Kea. SuZIE I.5 was able to achieve instrumentlimited performance ${ }^{21}$ down to $10 \mathrm{mHz}$ at $150 \mathrm{GHz}$ at the CSO by subtracting a combination of spatial and spectral commonmode signals (Mauskopf 1997). The initial subtraction of the spatial common-mode signal was obtained by differencing detectors separated by $\simeq 4^{\prime}$ and removed the atmospheric noise to within a factor of 2 of the instrument noise level below a couple

\footnotetext{
${ }^{21}$ For reference, SuZIE I.5's BLIP limit was a factor of $\simeq 3$ below the instrument noise limit at $100 \mathrm{mHz}$ and a factor of $\simeq 6$ below the instrument noise limit at $10 \mathrm{mHz}$.
} 

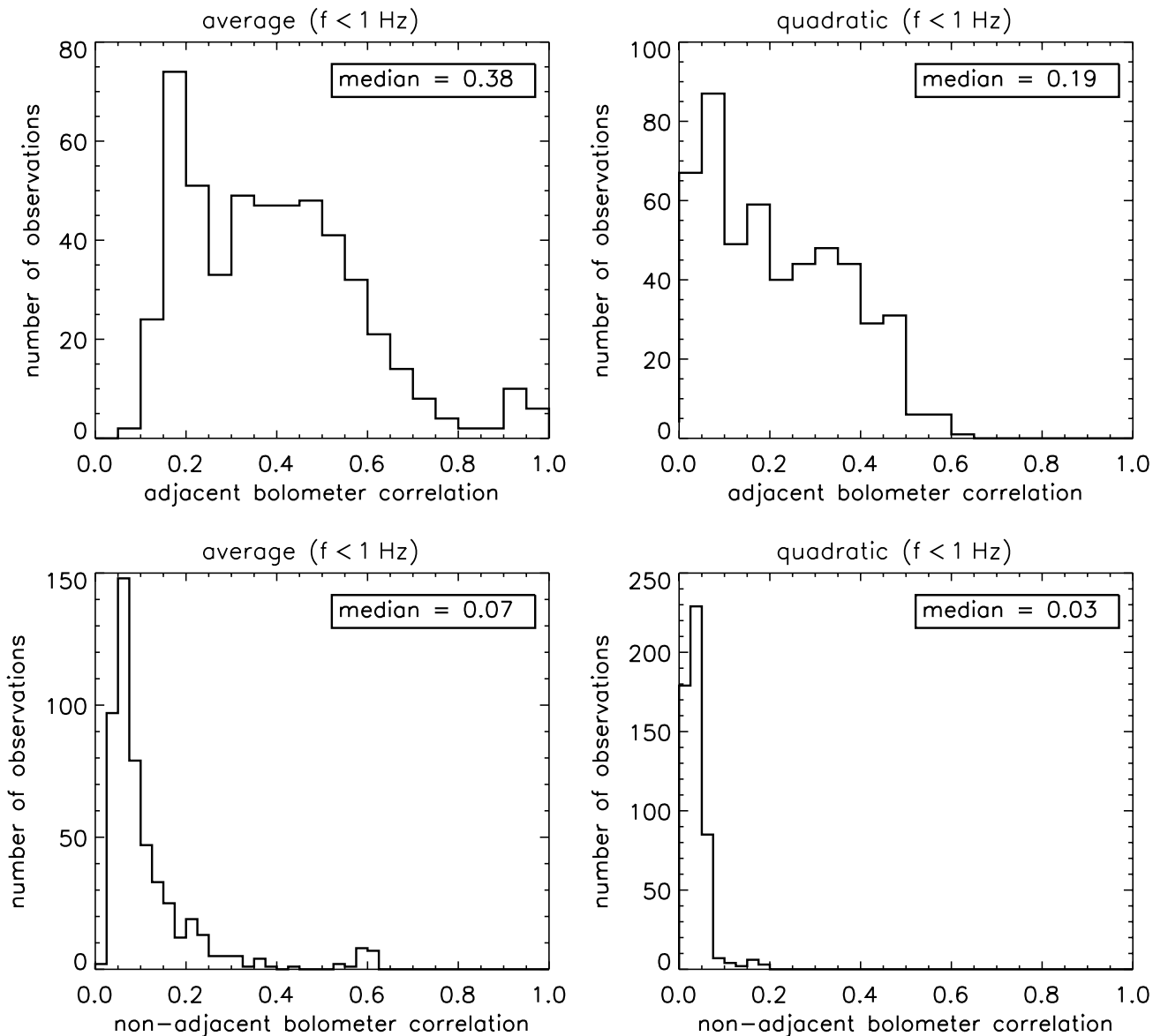

Figure 13. Histograms of the magnitude of the bolometer-bolometer correlations at frequencies below $1 \mathrm{~Hz}$ for both adjacent and non-adjacent bolometer pairs at $143 \mathrm{GHz}$. The plots on the left show data processed with average subtraction, and the plots on the right show data processed with quadratic subtraction. The top row shows adjacent bolometer correlations, and the bottom row shows non-adjacent bolometer correlations. Quadratic subtraction removes almost all of the atmospheric noise from the data; the residual atmospheric noise in the average subtracted data is the reason for the much higher correlations compared to quadratic subtracted data. Adjacent bolometers are still significantly correlated even after quadratic subtraction, this correlation is primarily due to the excess low-frequency noise described in Section 5.1.

hundred $\mathrm{mHz}$. In addition, SuZIE I.5 had three observing bands $(143,217$, and $269 \mathrm{GHz})$ per spatial pixel, which allowed determination of the correlated signal over a range of frequencies. The remaining atmospheric noise at low frequency was removed down to the instrument noise level by subtracting this spectral common-mode signal.

SuZIE II was able to employ a similar subtraction method, using observing bands at 143,221 , and $355 \mathrm{GHz}$ for each spatial pixel (Benson 2004). Additionally, SuZIE II had a much lower instrument noise level at $150 \mathrm{GHz}$ compared to SuZIE I.5, within $50 \%$ of the BLIP limit. Similar to Bolocam, SuZIE II reached the instrument noise level at frequencies above a couple hundred $\mathrm{mHz}$ by subtracting a spatial common-mode signal. However, by subtracting the spectral common-mode signal, SuZIE II achieved instrument noise limited performance below $100 \mathrm{mHz}$, and was within a factor of 1.5 of the instrument noise limit at $10 \mathrm{mHz}$. Therefore, spectral subtraction of the atmospheric noise does provide a method to achieve nearly BLIP performance from the CSO. The MKIDCam CSO facility camera, due to be deployed in 2010, will make use of these lessons; it will have 576 pixels each sensing four colors, thus providing the ability to perform both spatial and spectral subtraction of the atmospheric noise (Glenn et al. 2008).

Additionally, scanning the telescope more quickly can increase the amount of astronomical signal band that is free from

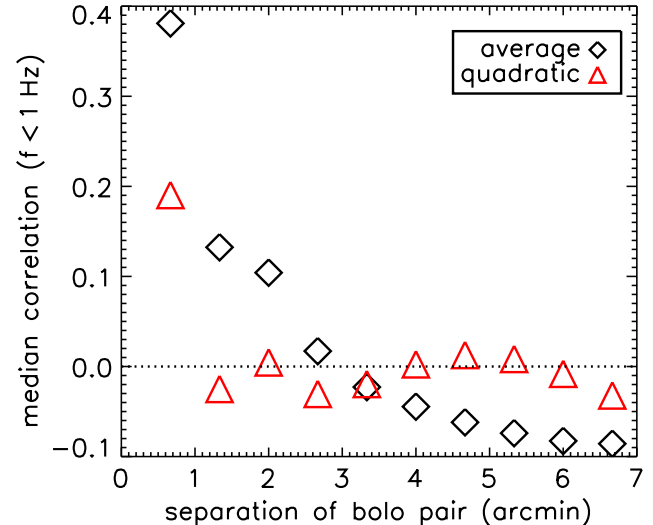

Figure 14. Plots of median bolometer-bolometer correlation fraction as a function of bolometer separation for time-stream data below $1 \mathrm{~Hz}$. The data have been averaged over all bolometer pairs and all $143 \mathrm{GHz}$ observations. The residual atmospheric noise can be easily seen in the average subtracted data as an excess correlation at small separations and an excess anti-correlation at large separations. In contrast, there is very little residual correlation in the quadratic subtracted data for non-adjacent bolometers, indicating that the atmospheric noise can be removed quite well with quadratic subtraction. The large spike in the correlation for adjacent bolometers is due to the excess low-frequency noise described in Section 5.1.

(A color version of this figure is available in the online journal.) 

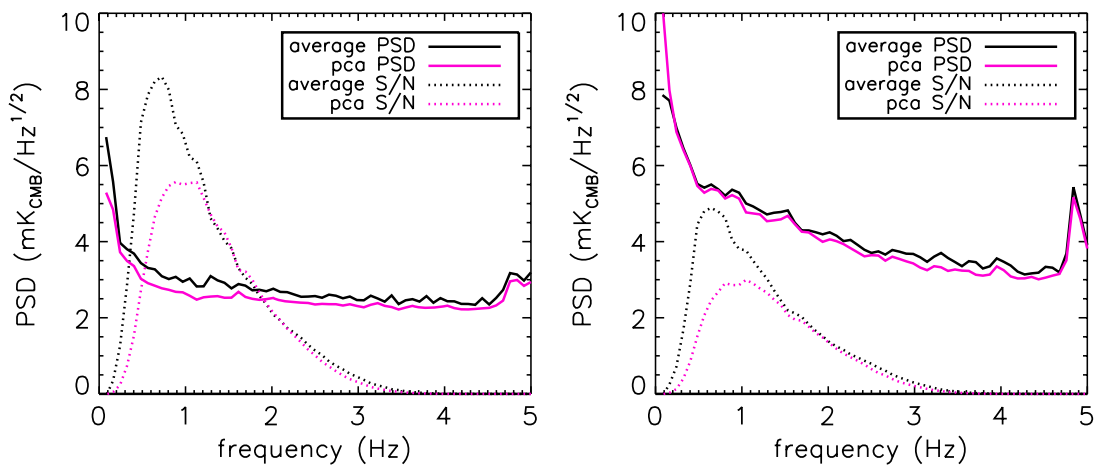

Figure 15. Plots of $143 \mathrm{GHz}$ time-stream PSDs averaged over all scans and all bolometers for a single observation for average subtraction and PCA subtraction. The left plot shows data from an observation made in relatively good weather, and the right plot shows an observation made in relatively poor weather. Overplotted as dotted lines is the $\mathrm{S} / \mathrm{N}$ for each subtraction method (in arbitrary units), calculated by dividing the window function for a CMB shaped signal by the noise PSD. Note that the $\mathrm{S} / \mathrm{N}$ is significantly higher for average subtraction compared to PCA subtraction at low frequencies because average subtraction attenuates much less signal. (A color version of this figure is available in the online journal.)

atmospheric noise. As long as the telescope scan speed is slower than the angular wind speed of the turbulent layer, the atmospheric noise power spectrum will remain unchanged in the time-stream data as the telescope scan speed is increased. For Bolocam at the CSO, this means that the atmospheric noise will remain below $\simeq 0.5 \mathrm{~Hz}$ for scan speeds below the average angular wind speed of $\simeq 30 \operatorname{arcmin~s}^{-1}$. Increasing the scan speed for Bolocam observations from 2-4 $\operatorname{arcmin~s} \mathrm{s}^{-1}$ to $30 \operatorname{arcmin~s}{ }^{-1}$ would increase the half-width of the beam profile from $\simeq 1-2 \mathrm{~Hz}$ to $\simeq 10-20 \mathrm{~Hz}$, significantly increasing the amount of astronomical signal band that is at frequencies above the atmospheric noise. Unfortunately, we are not able to collect Bolocam data at these fast scan speeds because it is impossible/inefficient to scan the CSO telescope faster than a few $\operatorname{arcmin~s}^{-1}$ (see footnote 9).

\section{RESIDUAL TIME-STREAM CORRELATIONS}

\subsection{Adjacent Bolometer Correlations}

There is a large excess correlation, above what is predicted by the K-T model of the atmosphere, between the time-streams of adjacent bolometers for $143 \mathrm{GHz}$ Bolocam observations. This excess correlation appears mainly at low frequencies in the timestream data $(f \leqslant 1 \mathrm{~Hz})$, and can be seen in the data in both of the following ways: (1) a residual offset between the correlation value for adjacent bolometers and the K-T model (see Figure 5); and as (2) a non-zero fractional correlation between adjacent bolometers after subtracting most of the atmospheric noise (see Figures 13 and 14 and Table 2). On average, this excess correlation between adjacent bolometers is $\simeq 2 \mathrm{mK}^{2}$ for $f \leqslant$ $1 \mathrm{~Hz}$. However, the amount of excess correlation depends on the amplitude of the atmospheric fluctuations; when the observations are sorted by the value of $B_{v}^{2}$, the average excess correlation in the lowest quartile is $\lesssim 1 \mathrm{mK}^{2}$, and the average excess correlation in the highest quartile is $\gtrsim 5 \mathrm{mK}^{2}$ (see Table 2). Since the amplitude of this excess correlation depends on the value of $B_{v}^{2},{ }^{22}$ and since it has a rising spectrum at low frequency, ${ }^{23}$ the source of this correlated noise appears to be atmospheric fluctuations.

\footnotetext{
22 At $143 \mathrm{GHz}$, the total optical load is almost independent of atmospheric conditions because most of the load is not sourced by the atmosphere (see Section 1.1). Therefore, there will only be a very weak correlation between $B_{v}^{2}$ and the amount of photon noise.

${ }^{23}$ The Bolocam electronics noise is white down to $\lesssim 10 \mathrm{mHz}$, so the only noise in the time-stream data with a rising spectrum at low frequency is the atmospheric noise.
}

In addition to the excess correlation between adjacent bolometers, there is also excess noise in the bolometer timestreams at low frequencies. After accounting for the electronics noise, photon noise, and atmospheric noise, there is an excess of $\simeq 4 \mathrm{mK}^{2}$ for $f \leqslant 1 \mathrm{~Hz}$. This excess noise increases when the value of $B_{v}^{2}$ increases, so it also appears to be sourced by the atmosphere, and thus we interpret it as excess correlation at zero spacing that should be considered together with the excess correlation between adjacent bolometers. Given this $\simeq 4 \mathrm{mK}^{2}$ of excess low-frequency time-stream noise, we speculate that the $\simeq 2 \mathrm{mK}^{2}$ of excess correlated noise between adjacent detectors is explained by the fact that adjacent detectors are separated by less than the smallest possible size of a spatial mode of the electromagnetic (EM) field that propagates through the optical system and arrives at the focal plane. ${ }^{24}$ The $143 \mathrm{GHz}$ Bolocam optics provide a detector spacing of $0.7(f / \#) \lambda$, compared to the diffraction spot size of $\simeq(f / \#) \lambda$, which means there will be significant correlations in the signal recorded by adjacent detectors. Using the optical properties of the telescope and Bolocam optics, along with the geometry of the focal plane, we calculated the amount of correlation between adjacent bolometers for a beam-filling source (like the atmosphere). The result is that approximately $50 \%$ of the $143 \mathrm{GHz}$ power received by adjacent bolometers is completely correlated, which is what we observe in this excess low-frequency noise.

Although this excess noise appears to be caused by atmospheric fluctuations, we do not have an adequate model to explain its source. The excess noise appears in single detector time-streams (along with adjacent detectors for the reasons argued above), which means it must be localized to a single beam. Additionally, since the noise appears at low frequencies in the time-streams, it must be sourced by fluctuations larger than $2^{\prime}-4^{\prime} \cdot{ }^{25}$ But, the Bolocam beams for adjacent pixels are only separated by $40^{\prime \prime}$; dozens of pixels are separated by less than $2^{\prime}-4^{\prime}$. Therefore, fluctuations with an angular size of $2^{\prime}-4^{\prime}$ will cause correlations between a large number of Bolocam detectors, not just adjacent ones. An alternate explanation is

\footnotetext{
${ }^{24}$ This fact is a consequence of the spatial coherence of the EM field from classical electromagnetism. It is interesting to note that the same effect holds for photon noise in addition to atmospheric noise, since pixels separated by $\lesssim(f / \#) \lambda$ form an intensity interferometer of the kind first discussed by Hanbury Brown \& Twiss $(1956,1957,1958)$. Therefore, atmospheric noise and photon noise (both the shot noise and wave noise terms) will be correlated for pixels separated by $\lesssim(f / \#) \lambda$. We discuss this correlated photon noise below. 25 Since the telescope scan speed is $2-4 \operatorname{arcmin~s}^{-1}$, noise appearing below $1 \mathrm{~Hz}$ must be sourced by modes larger than $2^{\prime}-4^{\prime}$.
} 
Table 2

$143 \mathrm{GHz}$ Data, $f<1 \mathrm{~Hz}$

\begin{tabular}{lccccc}
\hline \hline \multicolumn{1}{c}{ Parameter } & Bin 1 & Bin 2 & Bin 3 & Bin 4 \\
\hline$B_{v}^{2}\left(\mathrm{mK}^{2} \mathrm{rad}^{-5 / 3}\right)$ & $46 \pm 2$ & $170 \pm 10$ & $580 \pm 20$ & $4000 \pm 400$ \\
Raw atmosphere $\left(\mathrm{mK}^{2}\right)$ & $77 \pm 11$ & $131 \pm 22$ & $310 \pm 60$ & $1060 \pm 150$ & $280 \pm 60$ \\
Adj. corr. noise $\left(\mathrm{mK}^{2}\right)$ & $0.8 \pm 0.1$ & $1.1 \pm 0.1$ & $1.9 \pm 0.2$ & $5.8 \pm 0.3$ \\
Adj. corr. fraction & $0.39 \pm 0.04$ & $0.52 \pm 0.04$ & $0.42 \pm 0.07$ & $0.53 \pm 0.03$ & $0.46 \pm 0.03$
\end{tabular}

Notes. Description of the excess low-frequency noise that appears in the $143 \mathrm{GHz}$ time-stream data and is likely sourced by the atmosphere. The first four columns give the median value, and uncertainty on the median value, of the data when they are binned as a function of the amplitude of the atmospheric fluctuations, $B_{v}^{2}$. The final column gives the median values, and uncertainties on the median values, for the full data set. From top to bottom, the rows give the value of $B_{v}^{2}$; the raw atmospheric noise below $1 \mathrm{~Hz}$ prior to subtraction; the excess correlated noise between adjacent detectors below $1 \mathrm{~Hz}$ after accounting for residual atmospheric noise, and correlated photon/white noise; and the correlation fraction between adjacent detectors below $1 \mathrm{~Hz}$ after accounting for the correlations expected from residual atmospheric noise and photon/white noise. The excess noise rises at low frequency and increases as a function of $B_{v}^{2}$, indicating that it is sourced by the atmosphere. Additionally, the excess noise is $\simeq 50 \%$ correlated between adjacent detectors; we hypothesize that this correlation is a consequence of the $0.7(f / \#) \lambda$ spacing between these detectors.

motivated by the fact that the median amount of excess lowfrequency noise $\left(\simeq 4 \mathrm{mK}^{2}\right)$ is much less than the total amount of atmospheric noise in the Bolocam data below $1 \mathrm{~Hz}\left(\simeq 240 \mathrm{mK}^{2}\right)$. Therefore, this excess noise could be explained by atmospheric fluctuations at a reasonable height if there is an optical nonideality that couples $1 \%-2 \%$ of the beam to the atmosphere in a manner that is uncorrelated across the array, excluding the adjacent bolometer correlations discussed above.

This excess correlated noise is difficult to remove because it is only correlated among bolometers that are close to each other on the focal plane. We have attempted to remove this noise by constructing localized templates using the data from a bolometer and the $\leqslant 6$ bolometers that are adjacent to it on the focal plane. We have removed these localized templates from the data both before and after applying our atmospheric noise removal algorithm to the data. Unfortunately, subtracting these templates from the data resulted in an unacceptable amount of signal attenuation, and not all of the locally correlated noise was removed.

Additionally, as a consequence of the Bolocam detector spacing, we expect the atmospheric photon noise will also be $\simeq 50 \%$ correlated between adjacent detectors. Since the photon noise has a white spectrum, these correlations will have a larger effect at high frequencies in the time-stream data where there is almost no contamination from atmospheric noise. For Bolocam, the median white noise of $5 \mathrm{mK}^{2} \mathrm{~Hz}^{-1}$ is composed of $2.5 \mathrm{mK}^{2} \mathrm{~Hz}^{-1}$ of detector plus electronics noise and $2.5 \mathrm{mK}^{2}$ $\mathrm{Hz}^{-1}$ of photon noise. At frequencies above $2.5 \mathrm{~Hz}$, well above the sky noise, the median correlation between adjacent bolometer time-streams is $5 \%$, which means the median correlated noise is $5 \times 0.05=0.3 \mathrm{mK}^{2} \mathrm{~Hz}^{-1}$. As mentioned above, EM-field overlap between adjacent pixels implies that $50 \%$ of the photon noise should be correlated, yielding an expectation of $1.3 \mathrm{mK}^{2}$ $\mathrm{Hz}^{-1}$ of correlated white noise, roughly 4 times the observed value.

We speculate that this deficit of correlation in the photon noise is explained by the fact that high-angle scattering to warm surfaces in the relay optics is the dominant source of optical loading. ${ }^{26}$ Such scattering does not necessarily preserve the correlation of the EM field between adjacent pixels in the way that it is preserved for the transmitted beam. The EM-field

\footnotetext{
26 Physical optics calculations with ZEMAX indicate that overillumination of the relay optics is negligible, and optical tests with a cold source indicate very high-angle scattering, not mirror spillover, produces most of our observed optical load.
}

correlations between adjacent pixels are only guaranteed to be preserved for the $10 \%$ of our optical loading that is received from the atmosphere via the transmitted beam. However, we caution that we have no positive evidence supporting this scattering hypothesis for the observed deficit of correlated photon noise between adjacent detectors.

Finally, our hypothesis of EM-field overlap between adjacent detectors implies that the atmospheric noise will also be $\simeq 50 \%$ correlated between adjacent detectors as a result of our spacing. However, since most of the fluctuation power in the atmosphere is at large scales, the atmospheric noise in these detectors is already highly correlated. Therefore, the excess adjacent bolometer correlations will only appear in the atmospheric noise that the $\mathrm{K}-\mathrm{T}$ model predicts will be uncorrelated (i.e., the difference between the $\mathrm{K}-\mathrm{T}$ model prediction for adjacent bolometers and bolometers with zero separation). The median amount of noise predicted by the $\mathrm{K}-\mathrm{T}$ model to be uncorrelated between adjacent bolometers is $\simeq 0.2 \mathrm{mK}^{2}$, which means there will be $\simeq 0.1 \mathrm{mK}^{2}$ of correlated noise between adjacent bolometers that is not predicted by the $\mathrm{K}-\mathrm{T}$ model. This means that the atmospheric noise will only cause an excess correlated noise signal of $\simeq 0.1 \mathrm{mK}^{2}$ between adjacent detectors.

In summary, there is an excess noise that appears at low frequencies in the Bolocam time-stream data. The amount of excess noise depends on the amplitude of the atmospheric fluctuations, and it is approximately $\simeq 50 \%$ correlated between adjacent detectors. We hypothesize that this correlation is due to the EM-field overlap engendered by the geometry of the optical system and the physical separation between adjacent detectors. The available evidence suggests that this excess noise is due to the atmosphere, but we emphasize that we do not have a physical model to explain it, nor do we have direct evidence for our EM-field overlap hypothesis.

\subsection{Sensitivity Losses Due to Residual Atmospheric Noise and Adjacent Bolometer Correlations}

Ideally, the noise in our data would be uncorrelated between bolometers and have a white spectrum. This is approximately what we would expect if instrumental or photon noise was the dominant source of unwanted signal in our data time-streams. However, our data contain a significant amount of noise with a rising spectrum at low frequency. Some of this noise is due to residual atmospheric noise, and some is due to the excess low-frequency noise described in Section 5.1. As mentioned in Section 5.1, the excess low-frequency noise (along with 


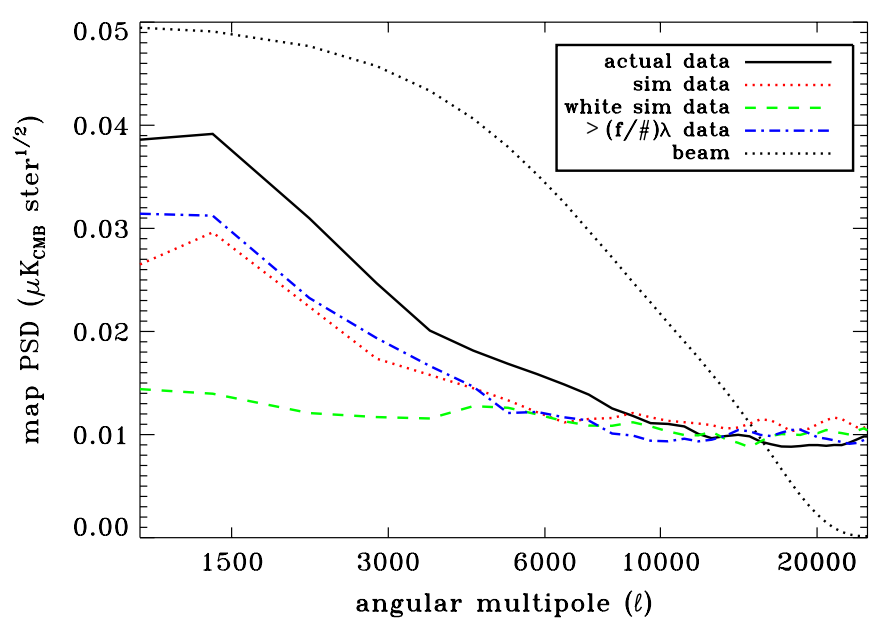

Figure 16. Map PSDs for actual and simulated time-streams. The solid black line shows the map PSD for all of the $143 \mathrm{GHz}$ Lynx data. The red dotted line shows the map PSD for simulated data generated using the noise spectrum of our actual time-streams, except that the simulated data are uncorrelated between detectors. The green dashed line shows the map PSD for uncorrelated simulated data that have a flat frequency spectrum and is based on the white noise level of our actual data. The blue dot-dashed line shows the map PSD for a map made from our actual data, after masking out some detectors so that the spacing between all detectors is $\gtrsim 1.3(f / \#) \lambda$. This reduces the number of detectors from 115 to 36 , but it discards the highly correlated data between adjacent detector pairs. Note that this spectrum has been multiplied by $\sqrt{36 / 115}$ to account for the change in the number of detectors. Since this PSD overlaps with the uncorrelated simulated PSD, we can conclude that most of the correlations between detector time-streams are among adjacent detector pairs, and these residual correlations have a significant impact on the noise of the resulting maps.

(A color version of this figure is available in the online journal.)

some residual atmospheric noise and photon noise) is highly correlated among adjacent bolometers. Additionally, there are correlations between all bolometer pairs on the focal plane due to the residual atmospheric noise. Finally, the atmospheric template used in our subtraction algorithms is constructed as a superposition of all the bolometer time-streams, so removing this template from each bolometer time-stream will cause it to be slightly correlated with every other bolometer time-stream.

To understand how these non-idealities affect our data, we have generated two sets of simulated data. A different simulated data set was generated for each detector for each $\simeq 10$-minutelong observation, based on the measured PSD of each bolometer for each observation. One simulated data set contains randomly generated data with the same noise PSD as our actual data, except the simulated data are completely uncorrelated between bolometers. The second set was generated using a flat noise spectrum (i.e., white noise), based on the white noise level observed in our actual data at high frequency. This simulated data set provides a best-case scenario for Bolocam. For each simulation, we generated data corresponding to all of the $143 \mathrm{GHz}$ observations of the Lynx science field, and the results are shown in Figure 16. Additionally, we made a map from our actual data after masking off 79 of the 115 detectors. This data set includes 36 detectors, all of which are separated by $\gtrsim 1.3(\mathrm{f} / \#) \lambda$, allowing us to test if the time-stream correlations are isolated to adjacent bolometer pairs. The results from this data set are also shown in Figure 16.

At high spatial frequency $(\ell \gtrsim 10000)$, the simulated data sets produce noise levels that are similar to our actual data, which implies that the correlations between detectors occur at low frequency and are caused by the atmospheric noise. However,
Table 3

Lynx Data

\begin{tabular}{lcc}
\hline \hline \multicolumn{1}{c}{ Data Type } & Data Spectrum & CMB Amplitude Uncertainty \\
\hline Actual data & Actual data & $270 \mu \mathrm{K}_{\mathrm{CMB}}^{2}$ \\
Simulated & Actual data & $170 \mu \mathrm{K}_{\mathrm{CMB}}^{2}$ \\
Simulated & White & $100 \mu \mathrm{K}_{\mathrm{CMB}}^{2}$ \\
Actual data, $>(f / \#) \lambda$ & Actual data & $170(550) \mu \mathrm{K}_{\mathrm{CMB}}^{2}$ \\
\hline
\end{tabular}

Notes. The estimated uncertainty on measuring the amplitude of a flat $\mathrm{CMB}$ power spectrum for all of the $143 \mathrm{GHz}$ Lynx observations. The four data sets include: our actual data, simulated data using our actual time-stream noise spectra, simulated data using our actual time-stream white noise level, and our actual data after masking off 79 of our 115 detectors so that the spacing between all detectors is $\gtrsim 1.3(f / \#) \lambda$. For the two simulated data sets, the bolometer time-streams are uncorrelated. The results for the second and fourth data sets are similar, after accounting for the reduction in detector number in the fourth set, indicating that the majority of the correlations between our detector timestreams are between adjacent detector pairs. The results show that our sensitivity to a CMB amplitude is reduced by a factor of $\simeq 1.6$ due to these correlations, and by another factor of $\simeq 1.7$ due to the residual atmospheric noise in our data at low frequencies.

both simulated data sets have a much lower noise level than our actual data at low spatial frequencies. To quantify the difference between the simulated data sets and our actual data set, we have estimated the uncertainty in determining the amplitude of a flat CMB power spectrum (see Sayers et al. (2009) for details of the calculation). Additionally, we estimated the uncertainty in determining the amplitude of a flat $\mathrm{CMB}$ power spectrum for the data set that contains our actual data for 36 detectors. This uncertainty was multiplied by $36 / 115$ to account for the degradation caused by masking off 79 detectors. The results are shown in Table 3. The simulated data indicate that our uncertainty on the amplitude of a flat $\mathrm{CMB}$ power spectrum would be improved by a factor of $\simeq 1.6$ if the detector timestreams were uncorrelated, and by another factor of $\simeq 1.7$ if the time-streams had a white spectrum instead of a rising spectrum at low frequency due to the residual atmospheric noise.

Additionally, after correcting for the loss of 79 detectors, the data set with 36 detectors produces a similar result to the simulated data set based on our actual noise spectra. This indicates that the correlations between time-streams of nonadjacent bolometers are negligible. The implication is that, if we had used larger horns (in $(f / \#) \lambda$ ) while maintaining the same number of detectors, we would have improved our sensitivity in $\mu \mathrm{K}_{\mathrm{CMB}}^{2}$ by a factor of 1.6. By going to larger horns, we would also have had a larger FOV, which would have had both positive (e.g., sensitivity to larger scales) and negative (e.g., less uniform map coverage) effects on our data. ${ }^{27}$ It seems likely that these negative effects would have been small compared to the large gain in sensitivity that we would have obtained by eliminating the excess correlations between adjacent bolometer time-streams. Another implication is that, at fixed detector count, it is more advantageous from the atmospheric noise point of view to use $\gtrsim(f / \#) \lambda$ pixel spacing and increase the FOV than it is to hold the FOV fixed and sample it more finely with $\lesssim(f / \#) \lambda$ pixel spacing. Increasing the Bolocam FOV was not

\footnotetext{
27 Additionally, there would be less correlation in the atmospheric noise signal over a larger FOV. However, given how well the K-T model describes the correlations as a function of separation in our data (see Figure 5), the correlation over an $8^{\prime}$ subregion of the FOV would be approximately equal to what we observed. Therefore, similar atmospheric noise removal could be obtained by performing the atmospheric noise subtraction algorithms on subregions of the larger FOV and/or subtracting higher order polynomials.
} 
possible by the time this effect was observed, but this lesson is being applied for MKIDCam.

\section{CONCLUSIONS}

We have studied the atmospheric noise above Mauna Kea at millimeter wavelengths from the CSO using Bolocam. Under all observing conditions, the data time-streams are dominated by atmospheric noise at frequencies below $\simeq 0.5 \mathrm{~Hz}$. The data are consistent with a $\mathrm{K}-\mathrm{T}$ turbulence model for a thin winddriven screen, and the median amplitude of the fluctuations is $280 \mathrm{mK}^{2} \mathrm{rad}^{-5 / 3}$ at $143 \mathrm{GHz}$ and $4000 \mathrm{mK}^{2} \mathrm{rad}^{-5 / 3}$ at $268 \mathrm{GHz}$. Based on a comparison to the ACBAR data in Bussmann et al. (2005), we conclude that these atmospheric noise fluctuation amplitudes are a factor of $\simeq 80$ larger than they would be at the South Pole for identical observing bands. This large difference in atmospheric noise amplitudes is due primarily to the South Pole being a much drier site than Mauna Kea, with a small factor of $\simeq 2$ arising from the fact that the fractional fluctuations in the column depth of water vapor are a factor of $\simeq \sqrt{2}$ lower at the South Pole. Based on our atmospheric modeling, we developed several algorithms to remove atmospheric noise, and the best results were achieved when we described the fluctuations using a low-order polynomial in detector position over the $8^{\prime}$ focal plane. However, even with these algorithms, we were not able to obtain BLIP performance at frequencies below $\simeq 0.5 \mathrm{~Hz}$ in any observing conditions. Therefore, we conclude that BLIP performance is not possible from Mauna Kea below $\simeq 0.5 \mathrm{~Hz}$ for broad band $\simeq 1-2 \mathrm{~mm}$ receivers with subtraction of a spatial atmospheric template on scales of several arcminutes. We also observed an excess low-frequency noise that is highly correlated between detectors separated by $\lesssim(f / \#) \lambda$; this noise appears to be caused by atmospheric fluctuations, but we do not have an adequate model to explain its source. We hypothesize that the correlations arise from the classical coherence of the EM field across a distance of $\simeq(f / \#) \lambda$ on the focal plane.

We acknowledge the assistance of the following: Minhee Yun and Anthony D. Turner of NASA's Jet Propulsion Laboratory, who fabricated the Bolocam science array; Toshiro Hatake of the JPL electronic packaging group, who wirebonded the array; Marty Gould of Zen Machine and Ricardo Paniagua and the Caltech PMA/GPS Instrument Shop, who fabricated much of the Bolocam hardware; Carole Tucker of Cardiff University, who tested metal-mesh reflective filters used in Bolocam; Ben Knowles of the University of Colorado, who contributed to the software pipeline, the day crew and Hilo staff of the Caltech Submillimeter Observatory, who provided invaluable assistance during commissioning and data-taking for this survey data set; high school teacher Tobias Jacoby and high school students Jonathon Graff, Gloria Lee, and Dalton Sargent, who helped as summer research assistants; and Kathy Deniston, who provided effective administrative support at Caltech. We thank W. L. Holzapfel for his many useful comments as referee of our manuscript. Bolocam was constructed and commissioned using funds from NSF/AST-9618798, NSF/AST-0098737, NSF/AST9980846, NSF/AST-0229008, and NSF/AST-0206158. J.S. and G.L. were partially supported by NASA Graduate Student Research Fellowships, J.S. was partially supported by a NASA Postdoctoral Program Fellowship, J.A. was partially supported by a Jansky Postdoctoral Fellowship, and S.G. was partially supported by a R. A. Millikan Postdoctoral Fellowship at Caltech. The research described in this paper was carried out at the Jet Propulsion Laboratory, California Institute of Technology, under a contract with the National Aeronautics and Space Administration.

Facility: CSO

\section{APPENDIX}

\section{APPENDIX MATERIAL}

In order to account for the time lags and advances between bolometer time-streams that are described by the K-T thinscreen model, we in general have to shift the time-streams by a fractional number of samples. For example, if a given bolometer time-stream is advanced by $\Delta t_{b}$ seconds, then we will account for this advance by shifting the time-stream according to

$$
d_{n}^{\prime}=\left(1-\left|\frac{\Delta t_{b}}{\Delta t}\right|\right) d_{n}+\left|\frac{\Delta t_{b}}{\Delta t}\right| d_{n+\Delta t_{b} / \Delta t},
$$

where $d_{n}^{\prime}$ is the interpolated data time-stream, $d_{n}$ is the original data time-stream, $\Delta t$ is the time between samples, and $n$ is the sample number. Note that we have assumed that $\Delta t_{b}<\Delta t$, since shifts by integer multiples of $\Delta t$ are trivial. Alternatively, this shift can be performed in frequency space by applying

$$
S_{m}=\left(1-\left|\frac{\Delta t_{b}}{\Delta t}\right|+\left|\frac{\Delta t_{b}}{\Delta t}\right| e^{-\operatorname{sign}\left(\Delta t_{b}\right) i 2 \pi f_{m} \Delta t}\right)
$$

to the Fourier transform of the time-stream data, where $f_{m}$ is frequency in $\mathrm{Hz}$ and $m$ is the frequency-space index. $S_{m}$ acts like a filter, and, for all non-zero frequencies, $\left|S_{m}\right|<1$. Therefore, to preserve the noise properties of our data, we divide the Fourier transform of the shifted time-stream by $\left|S_{m}\right|$. In summary, we shift the time-stream data according to Equation (1), then correct for the filtering effects of this shift in frequency space by dividing by $\left|S_{m}\right|$.

\section{REFERENCES}

Archibald, E. N., et al. 2002, MNRAS, 336, 1

Benson, B. A. 2004, PhD thesis, Stanford Univ.

Borys, C., et al. 1999, MNRAS, 308, 527

Bussmann, R. S., Holzapfel, W. L., \& Kuo, C. L. 2005, ApJ, 622, 1343

Chamberlin, R. A. 2004, PASA, 21, 264

Church, S. E. 1995, MNRAS, 272, 551

Conway, R. G., et al. 1965, MNRAS, 131, 159

Dobbs, M., et al. 2006, New Astr. Rev., 50, 960

Dowell, C. D., et al. 2003, Proc. SPIE, 4855, 73

Enoch, M., et al. 2006, ApJ, 638, 293

Glenn, J., et al. 1998, Proc. SPIE, 3357, 326

Glenn, J., et al. 2003, Proc. SPIE, 4855, 30

Glenn, J., et al. 2008, Proc. SPIE, 7020, 10

Haig, D. J., et al. 2004, Proc. SPIE, 5498, 78

Hanbury Brown, R., \& Twiss, R. Q. 1956, Nature, 178, 1046

Hanbury Brown, R., \& Twiss, R. Q. 1957, Proc. R. Soc. A, 242, 300

Hanbury Brown, R., \& Twiss, R. Q. 1958, Proc. R. Soc. A, 243, 291

Holland, W. S., et al. 1999, MNRAS, 303, 659

Jenness, T., et al. 1998, Proc. SPIE, 3357, 638

Kolmogorov, A. N. 1941, Dokl. Akad. Nauk SSSR, 30, 301

Kosowsky, A. 2003, New Astr. Rev., 47, 939

Kovacs, A. 2008, Proc. SPIE, 7020, 45

Kreysa, E., et al. 1998, Proc. SPIE, 3357, 319

Kreysa, E., et al. 2003, Proc. SPIE, 4855, 41

Lane, A. P. 1998, in ASP Conf. Ser. 141, Astrophysics from Antarctica, ed. G. Novak \& R. Landsberg (San Francisco, CA: ASP), 289

Laurent, G. T., et al. 2005, ApJ, 623, 742

Lay, O. P., \& Halverson, N. W. 2000, ApJ, 543, 787

Masson, C. R. 1994, in ASP Conf. Ser. 59, Astronomy with Millimeter and Submillimeter Wave Interferometry, IAU Colloquium 140, ed. M. Ishiguro \& J. Welch (San Francisco, CA: ASP), 87

Mauskopf, P. D. 1997, PhD thesis, Univ. California at Berkeley

Mauskopf, P. D., et al. 1997, Appl. Opt., 36, 765 
Murtagh, F., \& Heck, A. 1987, Multivariate Data Analysis (Boston: Kluwer) Pardo, J. R., Cernicharo, J., \& Serabyn, E. 2001a, IEEE Trans. Antennas Propag., 49, 1683

Pardo, J. R., Serabyn, E., \& Cernicharo, J. 2001b, J. Quant. Spectrosc. Radiat. Transfer, 68, 419

Pardo, J. R., et al. 2005, J. Quant. Spectrosc. Radiat. Transfer, 96, 537

Peterson, J. B., et al. 2003, Publ. Astron. Soc. Pac., 115, 383

Radford, S. J., \& Chamberlin, R. A. 2000, ALMA Memo, 334

Reichertz, L. A., et al. 2001, A\&A, 379, 735
Ruhl, J., et al. 2004, Proc. SPIE, 5498, 11

Sayers, J. 2007, PhD thesis, Caltech

Sayers, J., et al. 2009, ApJ, 690, 1597

Stark, A. A., et al. 2001, Publ. Astron. Soc. Pac., 113, 567

Tatarskii, V. I. 1961, Wave Propagation in a Turbulent Medium (New York: McGraw-Hill)

Taylor, G. I. 1938, Proc. R. Soc. A, 164, 476

Weferling, B., et al. 2002, A\&A, 383, 1088

Wright, M. C. H. 1996, Publ. Astron. Soc. Pac., 108, 520 